\title{
Formation of helium spectrum in solar quiescent prominences
}

\author{
N. Labrosse and P. Gouttebroze
}

\author{
Institut d'Astrophysique Spatiale, Bât. 121, Université Paris XI, 91405 Orsay Cedex, France \\ Received 14 August 2001 / Accepted 11 September 2001
}

\begin{abstract}
We present new non-LTE modelling of the helium spectrum emitted by quiescent solar prominences. The calculations are made in the frame of a one-dimensional plane-parallel slab. The physical parameters of our models are the electron temperature, the gas pressure, the slab width, the microturbulent velocity and the height above the solar surface. In this paper, we present isothermal isobaric models for a large range of temperature and pressure values. This work brings considerable improvements over the calculations of Heasley and co-workers (Heasley et al. 1974; Heasley \& Milkey 1976, 1978, 1983) with the inclusion in our calculations of partial redistribution effects in the formation of the $\mathrm{H}_{\text {I }} \mathrm{Ly} \alpha, \mathrm{Ly} \beta$, He I $\lambda 584 \AA$ and He II $\lambda 304 \AA$ lines. In addition we consider detailed incident profiles for the principal transitions. The statistical equilibrium equations are solved for a 33 bound levels (He I and HeII) plus continuum atom, and the radiative transfer equations are solved by the Feautrier method with variable Eddington factors. In this way we obtain the helium level populations and the emergent line profiles. We discuss the influence of the physical parameters on the helium level populations and on the main helium spectral lines. The effect of helium abundance in the prominence plasma is also studied. Some relations between singlet and triplet lines are given, as well as between optically thin or thick lines, He I and He II lines, and between the He I $\lambda 5876 \AA$ and H I $\lambda 4863 \AA$ lines. In a future work this numerical code will be used for the diagnostic of the prominence plasma by comparing the results with SUMER observations.
\end{abstract}

Key words. Sun: prominences - line: profiles - line: formation - radiative transfer

\section{Introduction}

Many non-LTE computations have been performed over more than thirty years in order to investigate radiative transfer effects and line formation in solar prominences. When one considers simple one-dimensional homogeneous and static models the first paper related to the helium spectrum in quiescent solar prominences which made satisfactory comparisons with observations is from Heasley et al. (1974, hereafter HMP). These authors removed several restrictive assumptions that were made before (see references in their paper). For example they used a multiion helium model atom with 20 energy levels and treated the radiative transfer problems in detail in the resonance lines and continua for the neutral and singly ionized helium. It was the first paper dealing with both hydrogen and helium spectra out of local thermodynamic equilibrium (NLTE). Their prominence models consisted of isobaric and isothermal slabs. Then a series of four papers with the same approach was published in the following years (Heasley \& Mihalas 1976; Heasley \& Milkey 1976, $1978,1983)$. These authors used detailed atomic models for both hydrogen and helium. However their work suffered from some limitations such as the assumption of

Send offprint requests to: N. Labrosse,

e-mail: labrosse@medoc-ias.u-psud.fr complete redistribution (CRD) in line formation, or the use of frequency-independent incident intensities for each line. They presented intensity ratios, integrated intensities and optical depths for several hydrogen and helium lines but no detailed emergent profile was shown.

Yakovkin et al. (1982) solved the integral diffusion equations for selected neutral helium levels with quantum number $n \leq 4$ under low-temperature plasma conditions and found that the excitation and ionization of helium are maximum near the boundaries. Other authors have considered different helium lines in different geometries in order to compare with specific observations. Morozhenko (1984) investigated the excitation of singlet helium levels in the frame of homogeneous and filamentary prominences. In a recent paper, Li et al. (2000) computed the He I $\lambda 5876 \AA$ (D3) emission line profile using the twocloud model and taking into account the multiplet. A work has been undertaken (Kucera et al. 1998; Andretta et al. 1999) in order to derive the neutral hydrogen column density and ionization state of helium from observations of hydrogen and helium continua.

In this paper we compute the essential parts of the neutral and ionized helium spectrum emitted by a model quiescent solar prominence. We use a plane-parallel slab standing vertically above the solar surface to represent the prominence. Computations allow departure from LTE for 
all atomic levels and the radiative transfer equations are solved for all lines and continua. Partial redistribution in frequency is included in the calculations of resonance lines. This represents a new step compared to the above-cited papers, towards a more physical approach. We obtain several radiative quantities related to the emergent spectrum. An important point is that we also get the emergent line profiles. This can be particularly useful for the interpretation of prominence spectra, especially when one observes optically thick lines such as the resonance lines of neutral (He I $\lambda \lambda 584$ and $537 \AA$ ) or ionized helium (He II $\lambda 304 \AA$ ) from which the derivation of the plasma physical parameters is not straightforward.

We explain in detail the approch used for the computations in Sect. 2. Then computed models similar to those of Heasley and co-workers are presented in Sect. 3 in order to compare them with previous theoretical results of HMP and Heasley \& Milkey (1978, hereafter HM3). The influence of the physical parameters of the models on the helium states mean populations and on the emerging spectrum is studied (Sect. 4) and some relations between helium line intensities and also the He I $\lambda 5876 \AA$ (D3) versus H I $\lambda 4863 \AA(\mathrm{H} \beta)$ relation are considered in Sect. 5 . In the conclusion, we indicate the future developments of this work.

\section{Modelling}

\subsection{Numerical procedure}

The first step in our calculations is to solve the ionization, hydrostatic and statistical equilibrium equations as well as the radiative transfer equations out of local thermodynamic equilibrium (non LTE) for hydrogen. Computational details can be found in Gouttebroze et al. (1993, hereafter GHV). We obtain electron and hydrogen level population densities from which we can determine the hydrogen spectrum emitted by our prominence. We thus derive the radiation inside the slab, taking into account the incident radiation and the principal transitions of the hydrogen atom. This defines the new physical conditions for the computation of the helium spectrum. The second step is then to solve independently the statistical equilibrium and the radiative transfer equations for the multilevel, multi-ion helium atom. The solution of the statistical equilibrium equations is determined by iterations and the radiative transfer equations are solved by the Feautrier method (finite-difference method, Feautrier 1964) with variable Eddington factors (Auer \& Mihalas 1970). In all calculations, partial redistribution in frequency is considered for the formation of the resonance lines H I Ly $\alpha$ and Ly $\beta$ (Heinzel et al. 1987), He I $\lambda 584 \AA$ and He II $\lambda 304 \AA$.

\subsection{Prominence model}

The prominence model used here is the same as described in GHV - see also Heinzel et al. (1987, 1994). It consists

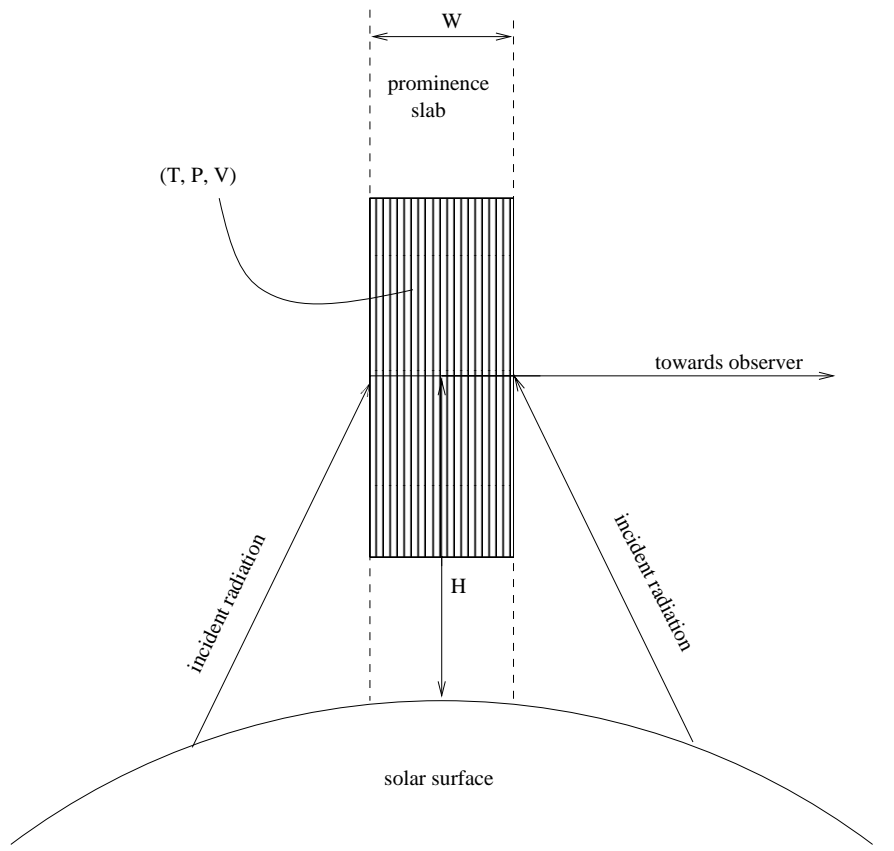

Fig. 1. Prominence model. $T, P$, and $V$ are respectively the electron temperature, the gas pressure, and the microturbulent velocity. $H$ is the height of observation above the solar surface and $W$ the slab geometrical thickness.

of a plane, parallel slab standing vertically above the solar surface (see Fig. 1). It is a one-dimensional representation defined by the slab thickness $W$. On both sides of this symmetric model, the prominence is illuminated by an incident radiation field coming from the underlying photosphere and chromosphere, and the surrounding corona. This radiation field determines the boundary conditions for the resolution of the radiative transfer equations and it is crucial to consider it properly in order to study line formation. This radiation field is diluted according to the height $H$ of the line-of-sight above the solar surface and eventually to the center-to-limb variations for the incident lines. In our computations the center-to-limb variations are taken into account for the incident hydrogen lines with upper level $n \leq 5$ (Gouttebroze \& Labrosse 2000). The emerging spectrum is computed for three viewing angles: $\mu=\cos \theta=0.2,0.6$ and 1 . This last value corresponds to a line-of-sight perpendicular to the slab and will be the only one considered in this paper, for brevity. Inside the slab, three physical quantities have to be defined: the electron temperature, the gas pressure and the microturbulent velocity. In this work we use isobaric and isothermal models, so these three quantities are fixed constant throughout the slab. Consequently, each model is defined by 5 parameters, namely the electron temperature, the gas pressure, the microturbulent velocity, the slab thickness and the height above the solar surface. 


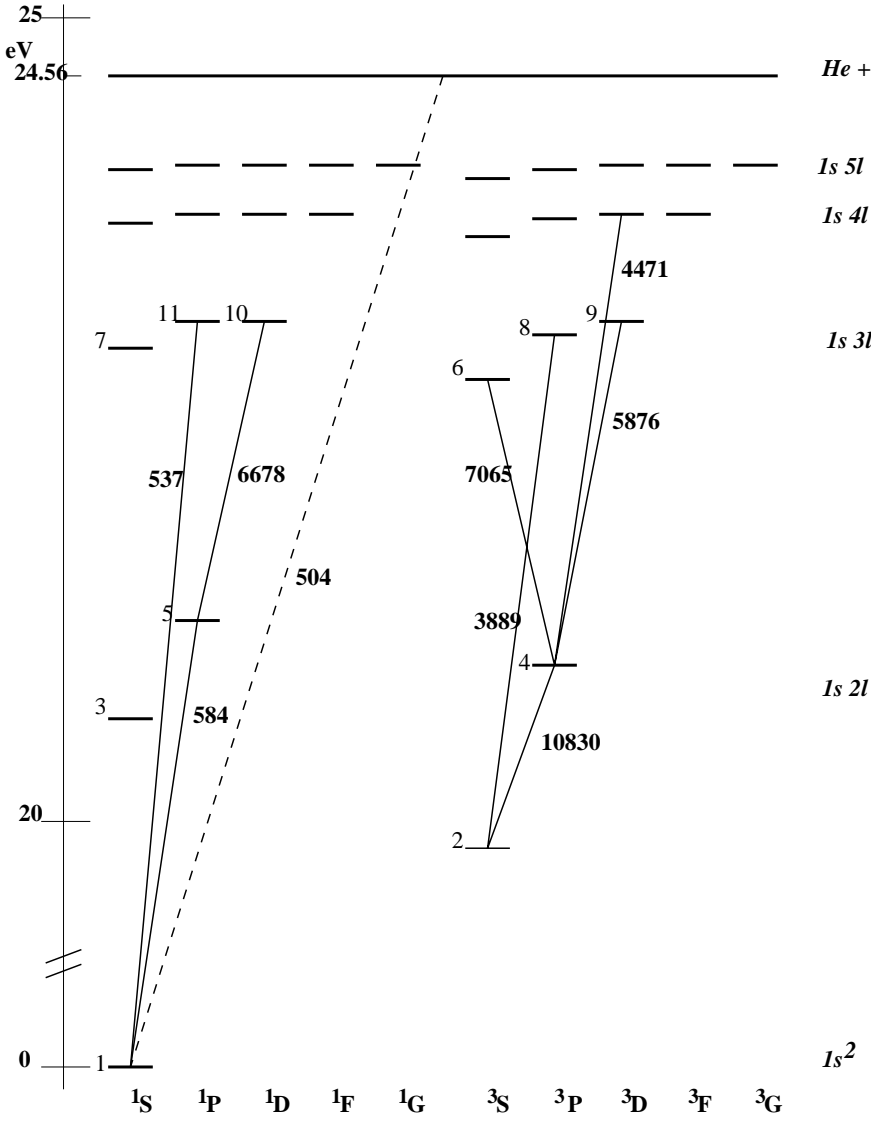

Fig. 2. Diagram of the neutral helium energy levels. The energy scale has been shortened between 0 and $20 \mathrm{eV}$. We indicate for the 11 first states the index of the level with increasing energy relative to the ground state. Solid lines represent some of the transitions often observed in quiescent prominences, and the dashed line represents the ionization continuum at $504 \AA$.

Numerical codes for the hydrogen emerging spectrum from a quiescent prominence can be found on the MEDOC web site ${ }^{1}$ (Gouttebroze \& Labrosse 2000).

\subsection{Atomic model}

We use very detailed atomic models for both hydrogen and helium. The hydrogen atom is the same as in GHV (20 bound levels plus continuum).

For neutral helium we use the atomic model of Benjamin et al. (1999, hereafter BSS). Thus we consider in our calculations 29 energy levels up to $n=5$, divided into individual $(L, S)$ states, which allows us to keep the distinction between singlet $(S=0)$ and triplet $(S=1)$ levels. Energy levels and statistical weights are given by Wiese et al. (1966). It is important to have this distinction in the model atom between neutral helium singlet and triplet levels because they are not populated through the same processes. Figure 2 represents schematically the neutral helium states included in the calculations up to $n=5$. Note that the energy scale has been modified in order to

\footnotetext{
1 http://www.medoc-ias.u-psud.fr/science/codes/ rtc.html
}

lessen the large gap between the ground state $1 \mathrm{~s}^{2}$ and the first excited level $1 \mathrm{~s} 2 \mathrm{~s}{ }^{3} \mathrm{~S}$. The latter is a metastable state and is the lower state of the infrared He I $\lambda 10830 \AA$ line. In Fig. 2 we show some line transitions which will be the subject of further investigations in Sects. 4 and 5, as well as the resonance continuum transition which occurs at $504 \AA$. The optically thick resonance lines lie in the singlet system. There are no permitted radiative transitions between the two systems, but they are coupled through collisions. Effective collision strengths, collisional ionization coefficients and spontaneous emission coefficients are from BSS. Collisions strengths not defined in BSS are taken in Benson \& Kulander (1972). Coefficients for Stark broadening are from Dimitrijevic \& Sahal-Brechot (1984), and from Griem (1974) for transitions not defined in Dimitrijevic \& Sahal-Brechot. Photoionization cross sections are from TOPBASE (Fernley et al. 1987).

For ionized helium we use a simple 4 bound levels atomic model. Energy levels and statistical weights again are given by Wiese et al. (1966). Effective collision strengths are from Aggarwal et al. (1992) for collisional transitions up to $n=3$ and from Aggarwal et al. (1991) for transitions up to $n=4$. Collisional ionization coefficients are calculated as in Mihalas \& Stone (1968). Spontaneous emission coefficients are those of Allen (1973). Photoionization cross sections are from TOPBASE.

The He III ion is represented by one level. With this He I-He II-He III system we treat in our computations 76 permitted radiative transitions and 438 collisional transitions.

\section{Comparisons with previous theoretical works}

It is interesting to compare our results to the pionnering work done by Heasley and his colleagues. We again emphasize that we have removed some restrictive simplifications that were made in their calculations such as the complete redistribution in frequency (CRD). Partial frequency redistribution (PRD) is considered in our calculations for the two first hydrogen Lyman lines and for HeI $\lambda 584 \AA$ and HeII $\lambda 304 \AA$. In addition, we use frequency-dependent incident line profiles for the principal transitions (see Table 1). Heinzel et al. (1987) have shown that in the case of hydrogen the combined use of these detailed incident profiles with PRD may change drastically the emitted profiles.

\subsection{Heasley, Mihalas, \& Poland (1974)}

The first paper of the series (HMP) considers hydrogen and helium (neutral and ionized) spectra emitted by the same geometrical prominence model as described above. The dilution factor for all lines and continua is taken to be $\frac{1}{2}$, corresponding to a zero altitude. They use a $15+4+1$ helium atom. Levels for He I with quantum number $n=4$ and $n=5$ are included as grouped $L$ states, keeping the distinction between singlet and triplet states. Collisional 
Table 1. Comparisons between modelling parameters for Heasley and co-workers and this work.

\begin{tabular}{|c|c|c|c|c|}
\hline & \multicolumn{2}{|r|}{ hydrogen } & \multicolumn{2}{|r|}{ helium } \\
\hline & Heasley et al. & here & Heasley et al. & here \\
\hline Number of levels & $5+1$ (HMP, HM2) & $20+1$ & $15+4+1$ & $29+4+1$ \\
\hline in the model atom & $35+1(\mathrm{HM} 3)$ & & & \\
\hline Frequency redistribution & & & & \\
\hline in resonance lines & complete (CRD) & partial (PRD) & complete (CRD) & partial (PRD) \\
\hline Detailed balance & Lyman lines & Lyman lines with $n>5$ & resonance lines & no \\
\hline Detailed incident profile & no & 10 lines with upper level $n \leq 5$ & no & 2 lines $(584 \AA, 304 \AA)$ \\
\hline
\end{tabular}

Table 2. Comparison of physical properties for HMP models. Units: electron temperature $T\left({ }^{\circ} \mathrm{K}\right)$; total hydrogen density $n_{\mathrm{H}}\left(\mathrm{cm}^{-3}\right)$. Population ratios are given at the surface and at the center of the slab.

\begin{tabular}{|c|c|c|c|c|c|c|c|c|c|c|}
\hline \multirow[b]{2}{*}{ Model } & \multirow[b]{2}{*}{$T$} & \multirow[b]{2}{*}{$n_{\mathrm{H}}$} & \multicolumn{4}{|c|}{ Optical Depth (total slab) } & \multicolumn{2}{|c|}{$n_{\mathrm{He} \mathrm{II}} / n_{\mathrm{He} \mathrm{I}}$} & \multicolumn{2}{|c|}{$n_{\text {Hе III }} / n_{\text {He II }}$} \\
\hline & & & $\tau^{912}$ & $\tau^{504}$ & $\tau_{0}^{584}$ & $\tau^{227}$ & surface & center & surface & center \\
\hline HMP 1 & 6000 & $10^{10}$ & $2.6+0$ & $3.8+0$ & $2.2+4$ & 8. -1 & 4.-1 & 3. -1 & 1. -4 & $8 .-5$ \\
\hline here & & & $1.1+1$ & $4.2+0$ & $2.8+4$ & 1. -1 & 3.-1 & 7.- -2 & 1. -2 & $9 .-3$ \\
\hline HMP 3 & 6000 & $10^{12}$ & $3.6+3$ & $1.0+3$ & $3.0+6$ & $1.5+2$ & 1.-2 & 1. -10 & 4. -6 & 1. -16 \\
\hline here & & & $3.9+3$ & $4.8+2$ & $3.1+6$ & 6. -3 & $2 .-2$ & 1. -11 & 4. -4 & 3. -6 \\
\hline HMP 7 & 8000 & $10^{10}$ & $2.3+0$ & $3.4+0$ & $1.7+4$ & $8 .-1$ & $5.3-1$ & $4.6-1$ & $2 .-4$ & 1. -4 \\
\hline here & & & $7.8+0$ & $3.8+0$ & $2.2+4$ & 1. -1 & $4.2-1$ & $1.1-1$ & 1. -2 & 1. -2 \\
\hline HMP 9 & 8000 & $10^{12}$ & $2.2+3$ & $8.8+2$ & $2.4+6$ & $1.3+2$ & $1 .-2$ & $3 .-7$ & 4. -6 & $3 .-16$ \\
\hline here & & & $1.9+3$ & $4.0+2$ & $2.2+6$ & $3 .-3$ & $2 .-2$ & $5 .-8$ & 4. -4 & 4. -6 \\
\hline
\end{tabular}

Table 3. Comparison of optical properties (HMP models). Integrated helium line intensities (ergs $\mathrm{cm}^{-2} \mathrm{~s}^{-1} \mathrm{sr}^{-1}$ ).

\begin{tabular}{rrr|rrrrrrrr}
\hline \multirow{2}{*}{ Model } & \multirow{2}{*}{$T$} & $n_{\mathrm{H}}$ & 304 & 537 & 584 & 3889 & 5876 & 6678 & 7065 & 10830 \\
\cline { 5 - 10 } & \multirow{2}{*}{$10^{10}$} & 341 & 8 & 356 & 235 & 1844 & 61 & 222 & 8438 \\
hMP 1 & 6000 & & 906 & 5 & 134 & 170 & 1300 & 21 & 164 & 6190 \\
\hline HMP 3 & \multirow{2}{*}{6000} & \multirow{2}{*}{$10^{12}$} & 132 & 4 & 54 & 73 & 567 & 6 & 69 & 2625 \\
here & & & 414 & 5 & 117 & 86 & 661 & 12 & 83 & 3140 \\
\hline HMP 7 & \multirow{2}{*}{8000} & \multirow{2}{*}{$10^{10}$} & 424 & 9 & 370 & 259 & 2032 & 61 & 245 & 9300 \\
here & & & 1100 & 6 & 154 & 197 & 1500 & 26 & 190 & 7160 \\
\hline HMP 9 & \multirow{2}{*}{8000} & \multirow{2}{*}{$10^{12}$} & 150 & 4 & 65 & 102 & 784 & 9 & 95 & 3615 \\
here & & & 431 & 6 & 134 & 60 & 449 & 20 & 56 & 2110 \\
\hline
\end{tabular}

rates are given by Mihalas \& Stone (1968), and Auer \& Mihalas (1973) give collisional rates not included in Mihalas \& Stone (1968) as well as photoionization rates. Oscillator strengths are obtained from Wiese et al. (1966). No turbulent broadening is considered, and CRD is assumed. HMP have adopted a relation which defines the radiation temperature as a function of wavelength for the incident radiation for continuum points (see their Fig. 1). But their relation was inaccurate for the continuum incident radiation shortward of $304 \AA$. They indeed extrapolated EUV fluxes for the He II resonance continuum from OSO-4 and OSO-6 spectrometers which had a wavelength cutoff at about $300 \AA$. In all our calculations we therefore use EUV fluxes given by Heroux et al. (1974) that give more ionizing radiation in the He II resonance continuum. No emergent profile is shown in their paper. Table 1 lists the main differences between their computations and our code.

We indicate in Tables 2 and 3 some comparisons for four computed models, which all have a slab thickness of $6000 \mathrm{~km}$ at height $H=0 \mathrm{~km}$, temperatures of 6000 (models HMP 1 and 3) and $8000 \mathrm{~K}$ (models HMP 7 and 9), and mean hydrogen densities of $10^{10}$ (models HMP 1 and 7 ) and $10^{12} \mathrm{~cm}^{-3}$ (models HMP 3 and 9). Table 2 presents 
the comparisons for the optical depths at the head of the Lyman continuum $\left(\tau^{912}\right)$, of the He I continuum $\left(\tau^{504}\right)$, of the He II continuum $\left(\tau^{227}\right)$, and at the 584 line center $\left(\tau_{0}^{584}\right)$, as well as the population ratios $n_{\mathrm{HeII}} / n_{\mathrm{HeI}}$ and $n_{\mathrm{He} \text { III }} / n_{\mathrm{He} \text { II }}$. Table 3 shows the results for the integrated intensities of the He II $\lambda 304 \AA$ line and several neutral helium lines.

One can note a rather good agreement between the two computations, except for the population ratios $n_{\mathrm{He} \text { III }} / n_{\mathrm{He} \text { II }}$ and $\tau^{227}$. At high densities a large disagreement occurs for those quantities. This discrepancy is mainly due to the different incident continuum radiation in the He II resonance continuum. We have an ionization continuum which is much more efficient to populate He III. Examination of $\tau^{504}$ and $\tau_{0}^{584}$ indicates that the neutral helium populations are of the same order in both works. At low densities we have less He II and more He III than HMP. The increase of pressure leads to lower $\tau^{227}$ in our computations but has the opposite effect in HMP calculations. This implies that the penetration of the EUV ionizing radiation is much more effective for our models. The result is that we get a much larger He III population in the slab. The integrated intensities in Table 3 reflect this situation. The neutral helium line intensities are roughly of the same order in HMP calculations and ours, but the He II $\lambda 304 \AA$ line intensity is systematically higher in our computations. At those high pressures fast recombinations from the He III ionization level follow the photoionization of the He II ground state. Radiative cascades towards the ground level of He II then occur, thus producing a strong emission in the 304 resonance line. Nevertheless the high densities of HMP models imply high pressures of about $1 \mathrm{dyn} / \mathrm{cm}^{2}$ and more which should not be regarded as very representative of the actual pressures in quiescent prominences.

\subsection{Heasley \& Milkey (1978)}

HM3 presented new computations of hydrogen and helium emerging spectra for more realistic models with more realistic temperature and pressure values as compared to HMP, new continuum incident intensities of Heroux et al. (1974), and lower dilution factors for the incident radiation. This was necessary for the authors in order to match the prominence observations made available by Landman \& Illing (1977). Unfortunately they do not give the new dilution factors that they adopted. We therefore use a dilution factor corresponding to an altitude of $10000 \mathrm{~km}$, viz. 0.416 (if there is no center-to-limb effect). CRD is still assumed for all lines and continua. Moreover they have solved the statistical equilibrium equations with the assumption that all Lyman lines are in detailed radiative balance. No emergent profiles and no quantities related to He II are given.

HM3 studied the triplet line D3 and the 6678/D3 singlet-triplet line ratio. Figure 3 presents comparisons between HM3 results and our computations for $E(\mathrm{D} 3)$
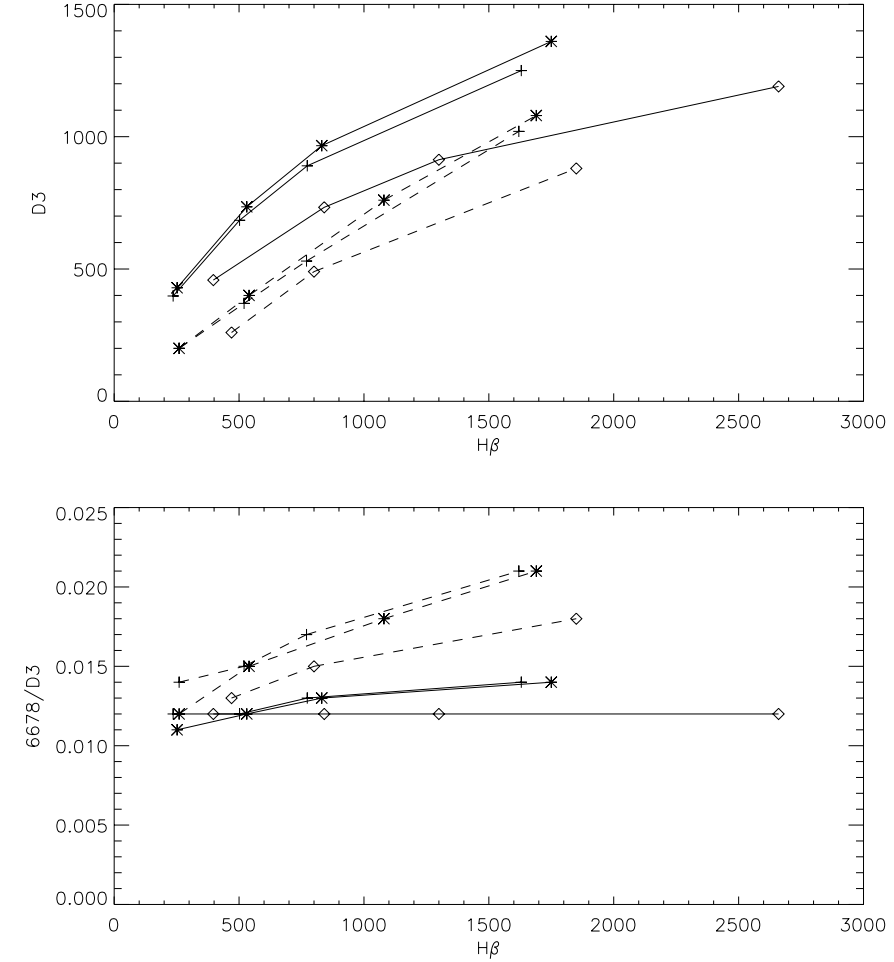

Fig. 3. $E(\mathrm{D} 3)$ (top) and $E(6678) / E(\mathrm{D} 3)$ (bottom) versus $E(\mathrm{H} \beta)$ for 3 models of HM3. Dashed lines are calculations by HM3, and solid lines represent our results. The three kinds of models are: 7500, $0.01(+)$; 9000, $0.015(*) ; 7500,0.02(\diamond)$. Integrated intensities are in cgs units.

and $E(6678) / E(\mathrm{D} 3)$ versus $E(\mathrm{H} \beta)$ for three classes of models without microturbulent velocity and defined by the temperature and the pressure: $(7500,0.01) ;(9000$, 0.015); (7500, 0.02) - see their Figs. 4 to 6 . In each model different calculations were made for column masses of $2 \times 10^{-6}, 4 \times 10^{-6}, 6 \times 10^{-6}$, and $1.2 \times 10^{-5} \mathrm{~g} / \mathrm{cm}^{2}$. We can notice that our $E(\mathrm{D} 3)$ are larger in every case than those of HM3. This seems to be due to a better penetration of the ionizing incident radiation in our models since helium recombination tends to populate the triplet levels. We also recall the uncertainty in the dilution factors that they used for the incident radiation. The relation between $E(\mathrm{D} 3)$ and $E(\mathrm{H} \beta)$ is studied in Sect. 5.5 for a larger number of models. On the other hand the ratio $E(6678) / E(\mathrm{D} 3)$ is lower in our computations than in HM3. Again the better penetration of the incident continuum explains this situation since ionization of helium hardly affects the singlet states populations but populates the triplet states through recombination. Moreover, the ratio $E(6678) / E(\mathrm{D} 3)$ varies much less in our computations, especially for the $(7500$, $0.02)$ models. It indicates that the line formation processes for both the D3 and the 6678 lines are not altered with the increase of the $\mathrm{H} \beta$ intensity, or in other words they do not change much with the increase of hydrogen column mass in this domain of temperatures and pressures. The main contribution in the line formation comes from the scattering of the incident radiation. Of course, absolute intensities increase with the hydrogen column mass. 
Table 4. Physical parameters adopted for the grid of 480 different prominence models.

\begin{tabular}{c|c|c}
\hline Parameter & Value & Unit \\
\hline Temperature & $6000,8000,10000,12000$, & \\
Pressure & $14000,16000,18000,20000$ & $\mathrm{~K}$ \\
Slab width & $0.02,0.05,0.10,0.20,0.50$ & $\mathrm{dyn} / \mathrm{cm}^{2}$ \\
Microturbulent & $200,1000,5000$ & $\mathrm{~km}$ \\
velocity & 5 & $\mathrm{~km} \mathrm{~s}^{-1}$ \\
Altitude & 10000 & $\mathrm{~km}$ \\
He abundance & & \\
$n_{\mathrm{He}} / n_{\mathrm{H}}$ & $0.05,0.10,0.15,0.20$ & \\
\hline
\end{tabular}

We will see in Sect. 5.3 (Fig. 18) that the relation between $E(6678)$ and $E(\mathrm{D} 3)$ strongly depends on the temperature and the pressure.

\section{Effect of physical parameters on helium states mean populations and on emergent intensities}

We have performed numerical calculations for both hydrogen and helium spectra for 480 models described in Table 4. In this section we show how the mean population densities of helium vary with the physical parameters of the prominence plasma. Mean populations are calculated following the formula:

$N_{i}=\frac{\int_{0}^{W} n_{i}(z) \mathrm{d} z}{W}$,

where $n_{i}(z)$ is the population of level $i$ at the depth $z$ in the slab, and $W$ is the slab width. We focus our attention on 5 neutral helium levels $\left(1 \mathrm{~s}^{2}, 1 \mathrm{~s} 2 \mathrm{p}{ }^{3} \mathrm{P}, 1 \mathrm{~s} 2 \mathrm{p}{ }^{1} \mathrm{P}, 1 \mathrm{~s} 3 \mathrm{~d}{ }^{3} \mathrm{D}, 1 \mathrm{~s} 3 \mathrm{~d}{ }^{1} \mathrm{D}\right.$, labelled $1,4,5,9$, and 10 in Figs. 4, 7 and 10), 2 ionized helium levels $(n=1$ and 2, labelled 30 and 31 ) and the continuum level $\mathrm{He}^{++}$ (labelled 34). Their mean populations are represented in Figs. 4, 7, and 10. Note that for clarity on those plots the mean populations of He I and He II ground states as well as the He III ionization level mean population have been multiplied by a factor $10^{-8}$. The bound levels chosen are involved in the transitions that we will particularly study in the following and in Sect. 5. We also present the influence of the different physical parameters presented in Table 4 on the emergent line profiles (Figs. 5, 8, and 11) and integrated intensities (Figs. 6, 9, and 12). The line profiles are for a normal emergent intensity (line-of-sight perpendicular to the slab surface). No result is shown concerning the influence of the microturbulent velocity and the height of the line-of-sight, because those parameters were taken as constant in the present study. We will more particularly consider four lines: He I $\lambda 584 \AA$ (optically thick resonance line between levels $1 \mathrm{~s}^{2}$ and $\left.1 \mathrm{~s} 2 \mathrm{p}{ }^{1} \mathrm{P}\right)$, He I $\lambda 6678 \AA$ (optically thin singlet line between $1 \mathrm{~s} 2 \mathrm{p}{ }^{1} \mathrm{P}$ and $\left.1 \mathrm{~s} 3 \mathrm{~d}{ }^{1} \mathrm{D}\right)$, He I $\lambda 5876 \AA$ (D3, optically thin triplet line
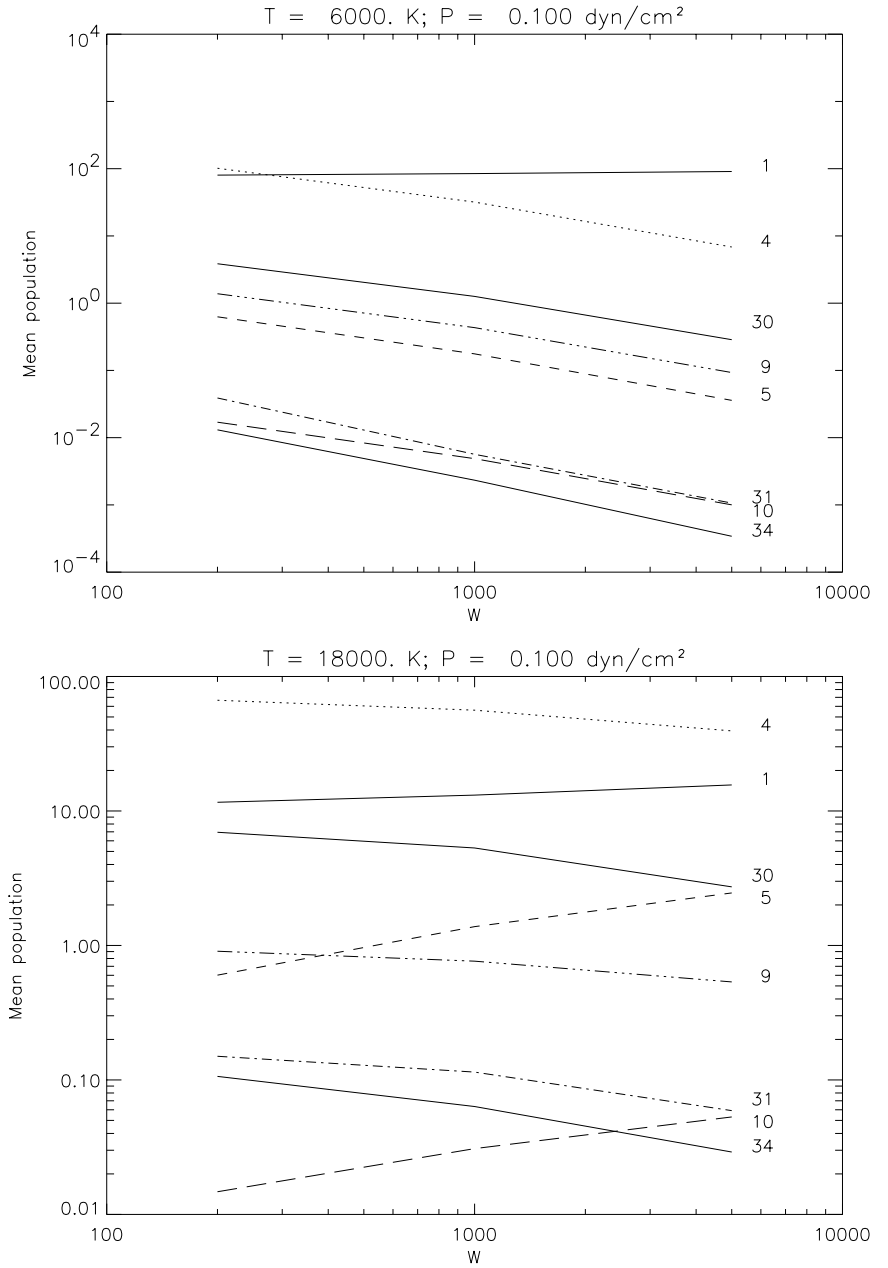

Fig. 4. Mean population densities $\left(\right.$ in $\mathrm{cm}^{-3}$ ) as a function of the slab width (in $\mathrm{km}$ ) for two different temperatures (6000 and $18000 \mathrm{~K})$ and one pressure $\left(0.1 \mathrm{dyn} / \mathrm{cm}^{2}\right)$. Solid lines: ground states of He I (1), of He II (30), and He III continuum (34). The population densities for these levels are divided by $10^{8}$. Singlet excited levels represented: $1 \mathrm{~s} 2 \mathrm{p}{ }^{1} \mathrm{P}(5$, dashes $)$ and $1 \mathrm{~s} 3 \mathrm{~d}{ }^{1} \mathrm{D}(10$, long dashes). Triplet levels are: $1 \mathrm{~s} 2 \mathrm{p}{ }^{3} \mathrm{P}\left(4\right.$, dots) and $1 \mathrm{~s} 3 \mathrm{~d}^{3} \mathrm{D}$ (9, long dashes/dots). Level 31 is the $n=2$ level of He II (short dashes/dots).

between $1 \mathrm{~s} 2 \mathrm{p}{ }^{3} \mathrm{P}$ and $1 \mathrm{~s} 3 \mathrm{~d}{ }^{3} \mathrm{D}$ ), and He II $\lambda 304 \AA$ (optically thick resonance line). This will give us an overview of the behaviour of the main helium spectral features in quiescent prominences.

\subsection{Influence of the slab width}

We can see the influence of the slab width in Figs. 4 to 6. At low temperatures the increase of the slab width tends to reduce the excited mean populations while the ground state mean population is almost constant. This is due to the fact that the ionizing radiation penetrates less deeply towards the slab center as the width increases. As a consequence the optically thick 584 line (which is mainly formed by the scattering of the incident radiation at that wavelength) does not show any sensitivity to the slab width. This is clearly seen in Fig. 5 (top panels) where we can 

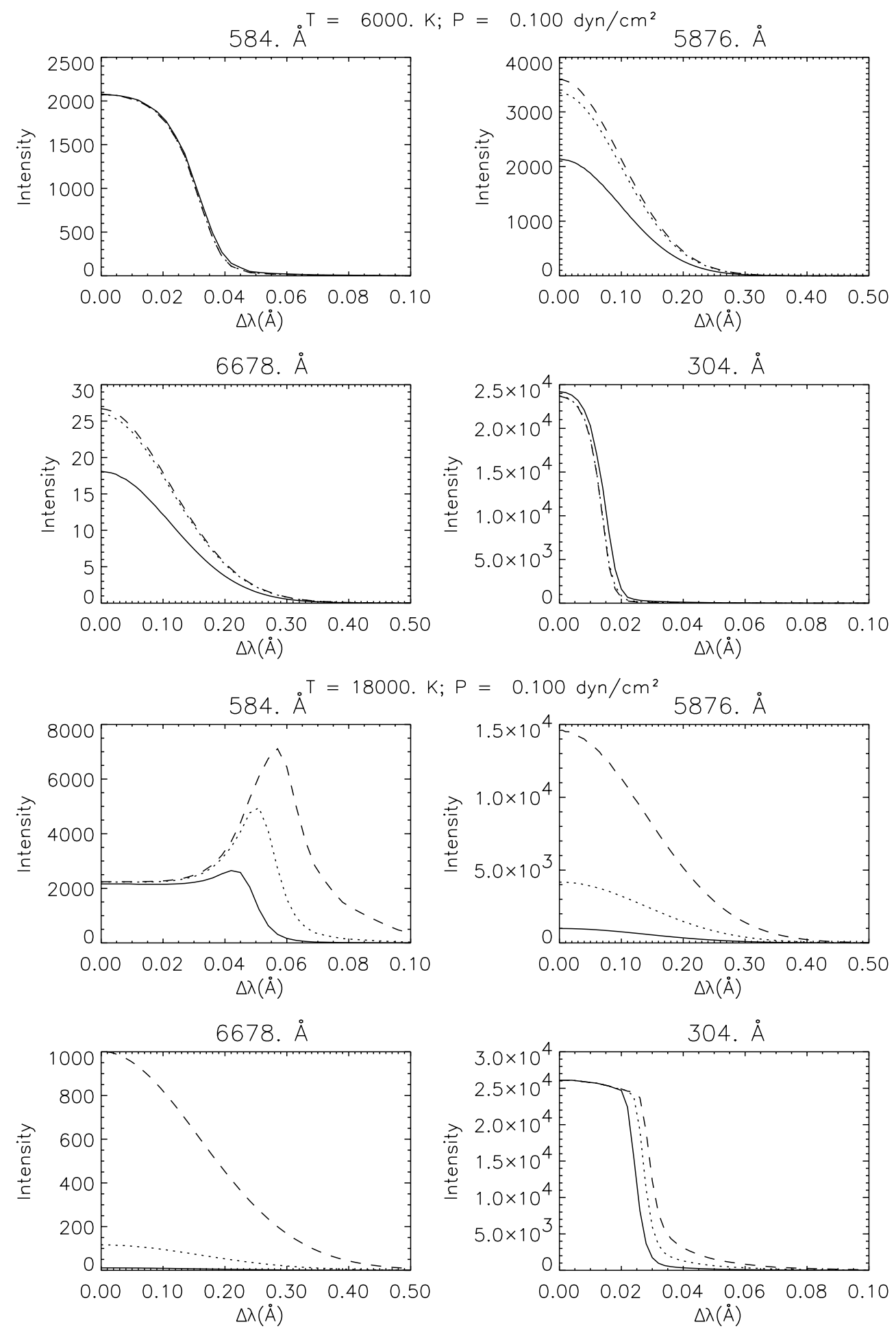

Fig. 5. Half emergent line profiles for one pressure $\left(0.1 \mathrm{dyn} / \mathrm{cm}^{2}\right)$ at $6000 \mathrm{~K}$ (four top panels) and $18000 \mathrm{~K}$ (four bottom panels) for three different slab widths. Solid line: $W=200$; dotted: $W=1000$; dashed: $W=5000 \mathrm{~km}$. Intensities are in $\operatorname{erg~s}^{-1} \mathrm{~cm}^{-2} \mathrm{sr}^{-1} \AA^{-1}$ (cgs units).

see that the line profile is not affected by the slab width variation. The optically thick 304 line is also not affected by the width change. One can even note a very slight decrease in the integrated intensity possibly due to continuous absorption in the core of the slab. On the contrary the optically thin lines are brighter when the slab width increases as a result of the increase of the optical thickness and, consequently, of the scattering of the incident radiation. However this brightening is not proportional to the width because the excitation of the lower levels of the transitions also depends on the penetration of the incident radiation in the resonance continuum at $504 \AA$ : the ionizing radiation penetrates less deeply as the slab width increases. This effect is visible in Fig. 5: the 

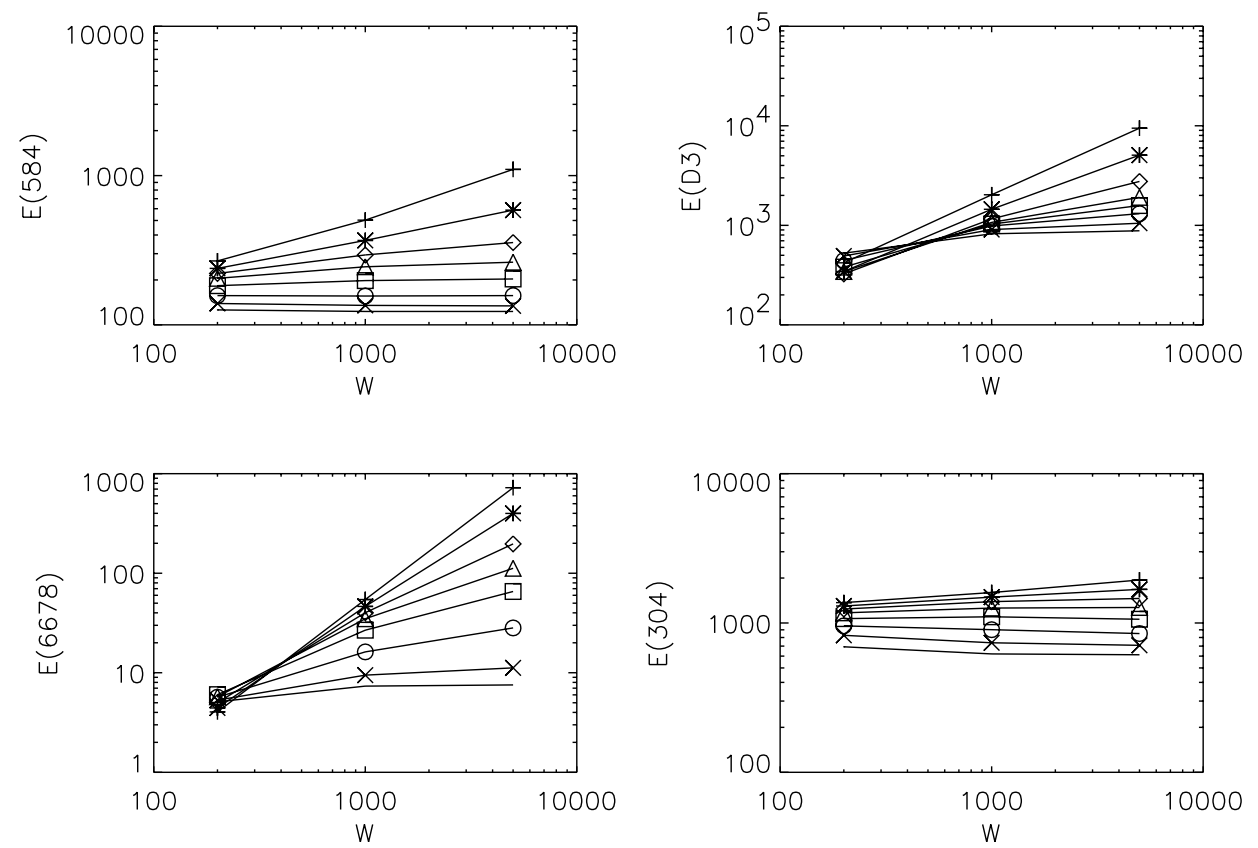

Fig. 6. Integrated intensities (in cgs units) for 4 lines as a function of the slab width $W$ (in $\mathrm{km}$ ) computed for one pressure $\left(0.1 \mathrm{dyn} / \mathrm{cm}^{2}\right)$ and 8 temperatures : $6000 \mathrm{~K}(\mathrm{no} \mathrm{symbol}), 8000 \mathrm{~K}(\times), 10000 \mathrm{~K}(\circ), 12000 \mathrm{~K}(\square), 14000 \mathrm{~K}(\triangle), 16000 \mathrm{~K}(\diamond)$, $18000 \mathrm{~K}(*)$, and $20000 \mathrm{~K}(+)$.

brightening of the emergent profile is larger when the slab width goes from 200 to $1000 \mathrm{~km}$ than between 1000 and $5000 \mathrm{~km}$.

At high temperatures the situation is quite different. The mean populations of He I ground state and singlet excited levels increase with the slab width while the mean populations of triplet levels of $\mathrm{He}$ I and He II decrease (Fig. 4, bottom panel). Again, the helium ionization decreases at slab center as the width increases. The triplet levels populations follow more or less the He II mean populations since the dominant population mechanism for the triplet system is the photoionization from the ground state of HeI, followed by recombinations to the triplet levels. This mechanism is known as the photoionization - recombination (PR) process and is the dominant population mechanism for helium below $20000 \mathrm{~K}$ (Andretta \& Jones 1997). However we can see in Fig. 5 (bottom panels) that the collisional excitations play an important role on the neutral helium lines formation. The optically thin lines are brighter at line center as well as in the wings. The 304 line is saturated at line center where only resonant scattering occurs but the wings are broadened when the slab width increases. As the 584 line, it is also saturated at line center but we observe an intensity peak between 0.05 and $0.06 \AA$ from line center due to collisional excitations. The total number of photons created by collisional excitation increases with the slab width. We can also note that the increase of the mean populations of neutral helium singlet levels with the slab width at high temperature is due to intersystem (triplet to singlet states) collisions. But in any case it is seen in Fig. 4 that the singlet states are underpopulated relative to their triplet equivalent states $\left(N_{5}<N_{4}\right.$ and $\left.N_{10}<N_{9}\right)$ whatever the temperature.

\subsection{Influence of the temperature}

The effect of the temperature on the mean populations and the emergent intensities are shown in Figs. 7 to 9 . At low pressures we can see that an increase of the temperature reduces the neutral helium mean population and raises the ionized helium population (top panel of Fig. 7). Moreover we note that at low pressures, for a temperature greater than $11000 \mathrm{~K}(\log (T)=4.05)$, we get $n_{\text {He II }}>n_{\text {He I }}$. The small optical thickness $(\tau \lesssim 1)$ of the Lyman continuum of hydrogen and of the neutral helium continuum favors the penetration of the EUV ionizing radiation. Since, at the same time, the recombination rates are low, we obtain a high helium ionization. The 584 line profile (Fig. 8) shows an increase of thermal emission in the line wings with the temperature, as the 304 line. At line center these optically thick lines are saturated and scatter the radiation. On the contrary the increase of temperature, which lowers the optical thickness, leads to a decrease of the scattering for the optically thin lines and thus a decrease of their emergent intensities (Fig. 9).

At high pressures all mean populations increase with the temperature except that of the He I ground state (bottom panel of Fig. 7). But now helium ionization is lower $\left(n_{\mathrm{He} \mathrm{II}}<n_{\mathrm{HeI}}\right)$ because of optical thickness of the neutral helium continuum $\left(\tau^{504}>1\right)$ which prevents the EUV incident radiation from reaching the core of the slab. The higher pressures also give higher recombination rates. All emergent intensities grow with temperature (Fig. 9). In Fig. 8 we see that in the 584 resonance line a peak at about $0.05 \AA$ from line center appears above $14000 \mathrm{~K}$, and its intensity increases with the temperature, while the line center is saturated. At high pressures collisional excitation becomes more significant when the temperature 

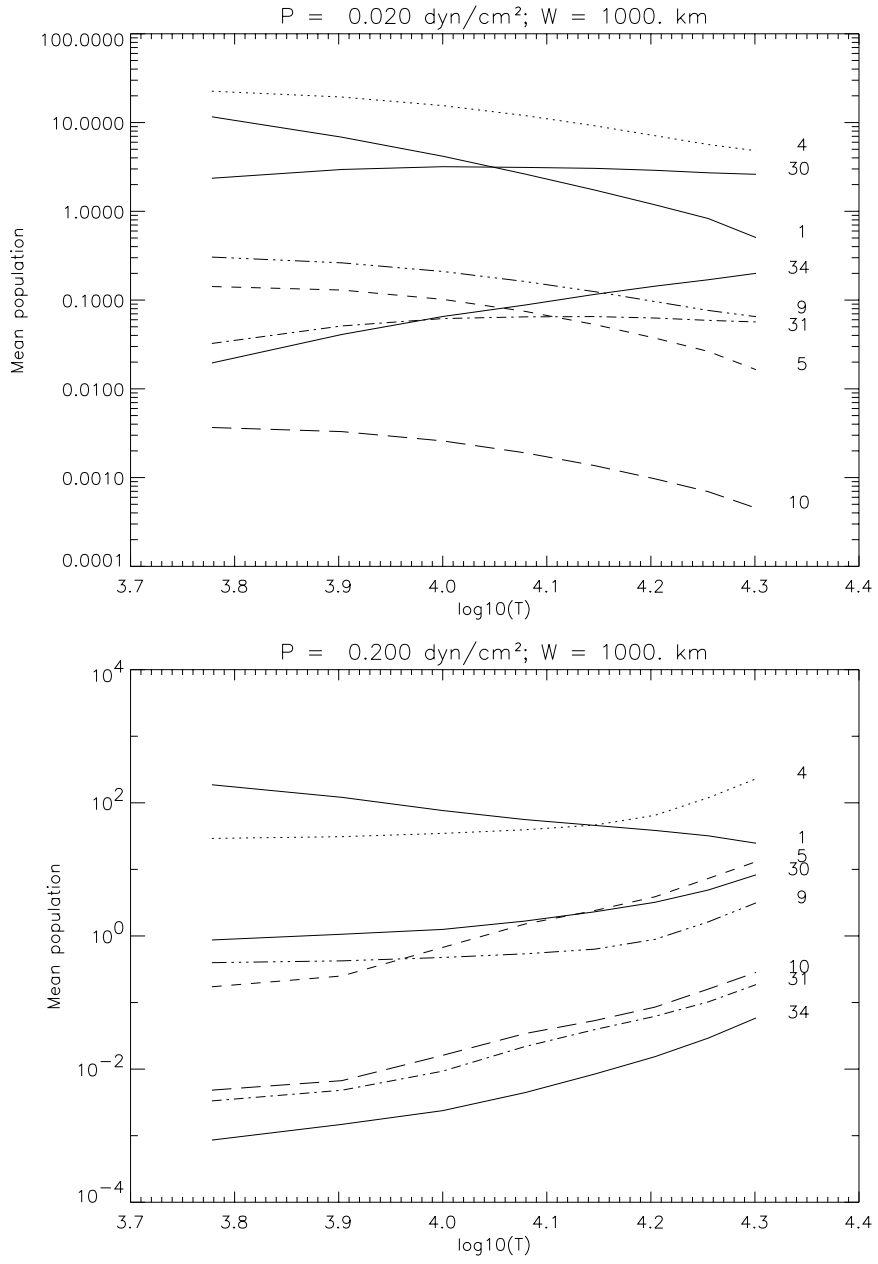

Fig. 7. Mean population densities $\left(\right.$ in $\mathrm{cm}^{-3}$ ) as a function of the temperature (in $\mathrm{K}$ ) for two different pressures (0.02 and $\left.0.2 \mathrm{dyn} / \mathrm{cm}^{2}\right)$ and one slab width $(1000 \mathrm{~km})$. Solid lines: ground states of He I (1), of He II (30), and He III continuum (34). The population densities for these levels are divided by $10^{8}$. Singlet excited levels represented: $1 \mathrm{~s} 2 \mathrm{p}{ }^{1} \mathrm{P}(5$, dashes $)$ and $1 \mathrm{~s} 3 \mathrm{~d}{ }^{1} \mathrm{D}(10$, long dashes). Triplet levels are: $1 \mathrm{~s} 2 \mathrm{p}{ }^{3} \mathrm{P}\left(4\right.$, dots) and $1 \mathrm{~s} 3 \mathrm{~d}{ }^{3} \mathrm{D}$ (9, long dashes/dots). Level 31 is the $n=2$ level of He II (short dashes/dots).

increases for this transition. For He II $\lambda 304 \AA$ the line center saturation is less pronounced than at low pressures but the temperature rise mostly broadens the profile. The 304 emitted intensity is not very sensitive to the temperature (Fig. 9), probably because the considered temperatures lie well below the excitation temperature of this line. From He II $\lambda 1640 \AA$ line profiles observed with Skylab in a prominence, Mariska et al. (1979) derived an average temperature of $27000 \mathrm{~K}$ for the region where He II is emitted. Our computed models have temperatures below $20000 \mathrm{~K}$ and the collisional processes are likely of secondary importance relative to the scattering of the incident radiation for the 304 line. Finally the optically thin lines at high pressures are mostly affected by thermal processes which enhances the line intensity as the temperature increases.

\subsection{Influence of the gas pressure}

The evolution of the mean populations, emergent line profiles and integrated intensities with the pressure can be seen in Figs. 10 to 12. At low temperatures the HeII and He III mean populations decrease with the pressure while a large increase occurs for the He I ground state population (Fig. 10, top panel). The populations of the excited singlet and triplet levels are roughly constant. The increase of the pressure raises the optical depth in the ionization continuum. Thus the helium ionization ratio decreases with pressure. Looking at the emergent profiles (Fig. 11) confirms this: the 584 line only scatters the incident radiation because of its large optical thickness and the pressure increase has almost no influence on the profile shape. Due to the large decrease of the ionization ratio and the increase of the continuum optical depth, the 304 line intensity is reduced. The increase in pressure produces an increase in collisional excitation in the optically thin lines.

At higher temperatures all neutral helium states see their mean populations increasing with pressure (bottom panel of Fig. 10). This is also the case for the He II populations but less markedly. He III mean population decreases with the pressure. At those high temperatures the optical depth at $912 \AA$ is less than unity. The helium ionization ratio in this case is larger than at low temperatures and recombination becomes more efficient as the pressure increases to populate the neutral helium excited levels. Moreover the 504 He I continuum becomes optically thick as the pressure increases and thus decreases the $n$ (He II $) / n$ (He I) and the $n$ (He III $) / n$ (He II) ratios. The optically thin line profiles show the same characteristics than at low temperature (see Fig. 11) but at high temperatures the collisional excitation enhances the line intensity. The 584 line also shows the importance of collisional excitation with a brightening in the wing giving an emission peak at about $0.05 \AA$ while line center is still saturated and only permits scattering of the incident radiation. At those high temperatures, collisional processes become non negligible in the formation of the line relative to the scattering of the incident radiation. This is obviously not the case for the 304 line where no intensity increase is observed (see also Fig. 12). As previously stated, the temperatures under consideration are not high enough to see any effect of collisional processes in the formation of the line and we only observe scattering of radiation.

\subsection{Influence of the helium abundance}

The helium abundance is a long-standing issue in the solar atmosphere as well as in prominences. Many authors have tried to determine the abundance from prominence observations and HM3 have used their modelling of $\mathrm{HI}$, He I and Ca II lines to infer a helium-to-hydrogen ratio of $0.10 \pm 0.025$. Lower ratios have been proposed by Yakovkin et al. (1982). These authors inferred a helium abundance close to 0.05 from a solution of the integral diffusion equations. However they only computed low-temperature 

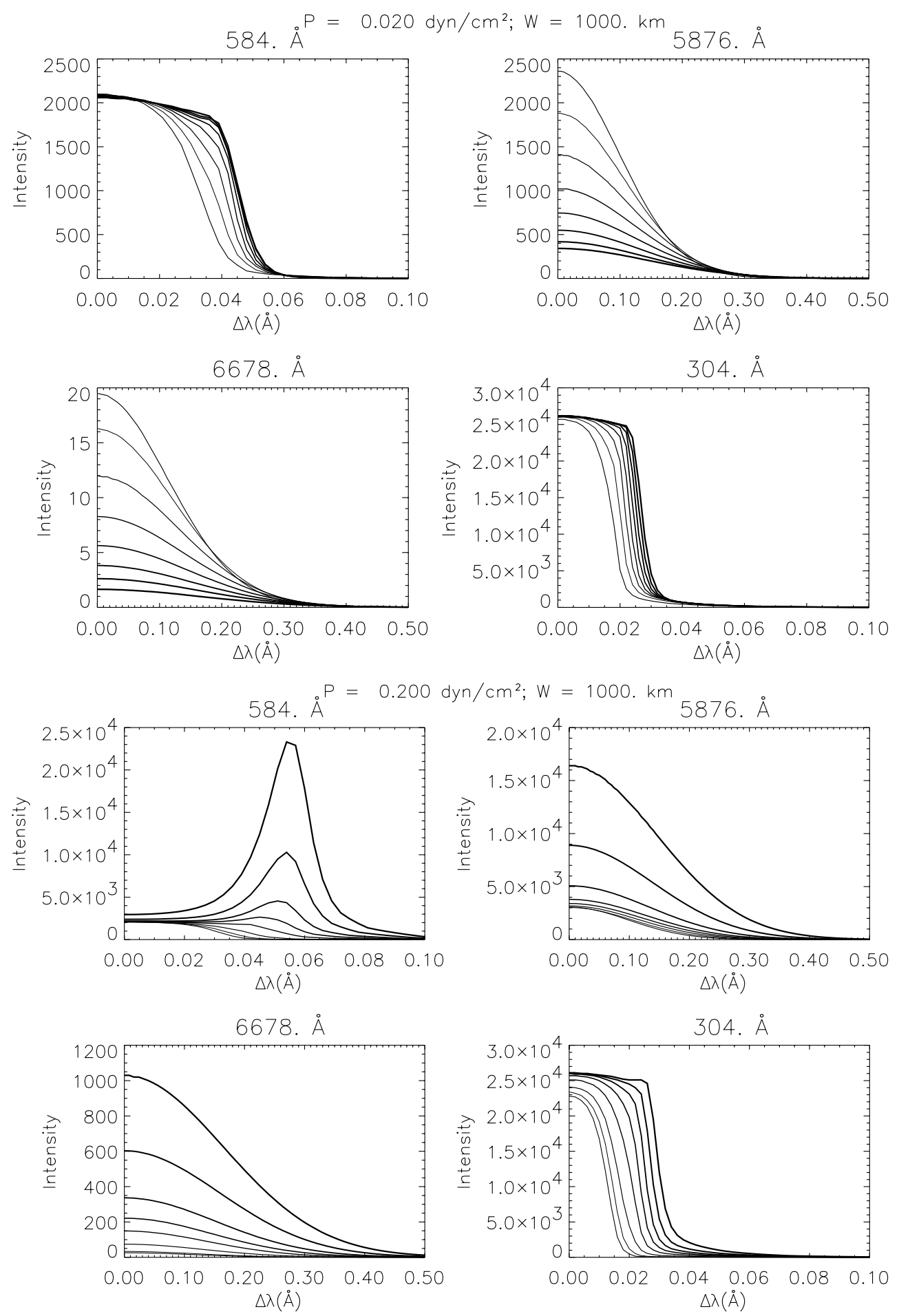

Fig. 8. Half emergent line profiles for one slab width $\left(1000 \mathrm{~km}\right.$ ) at $0.02 \mathrm{dyn} / \mathrm{cm}^{2}$ (four top panels) and $0.2 \mathrm{dyn} / \mathrm{cm}^{2}$ (four bottom panels). The increase of line thickness corresponds to an increase of temperature. The different temperatures are 6000 , 8000, 10000, $12000,14000,16000,18000$, and 20000 K. Same units as in Fig. 5.

models $\left(T_{\mathrm{e}}=7000 \mathrm{~K}\right)$ that do not take into consideration the presence at the prominence edges of hotter plasma. Hirayama (1971) found 0.065 with the assumption that both hydrogen and helium are fully ionized in the emitting region, an assumption that is unrealistic. In this paper we present theoretical calculations with a helium abundance of 0.10 by number relative to hydrogen. Nevertheless in this section we want to explore as a first step the influence of the helium abundance on mean populations, line profiles and integrated intensities. For the sake of simplicity we have chosen only two different models corresponding to different physical conditions in the prominence: model 6 , with $T=6000 \mathrm{~K}, P=0.02 \mathrm{dyn} / \mathrm{cm}^{2}$ and $W=1000 \mathrm{~km}$, and model 99 , with $T=18000 \mathrm{~K}, P=0.2 \mathrm{dyn} / \mathrm{cm}^{2}$ and 

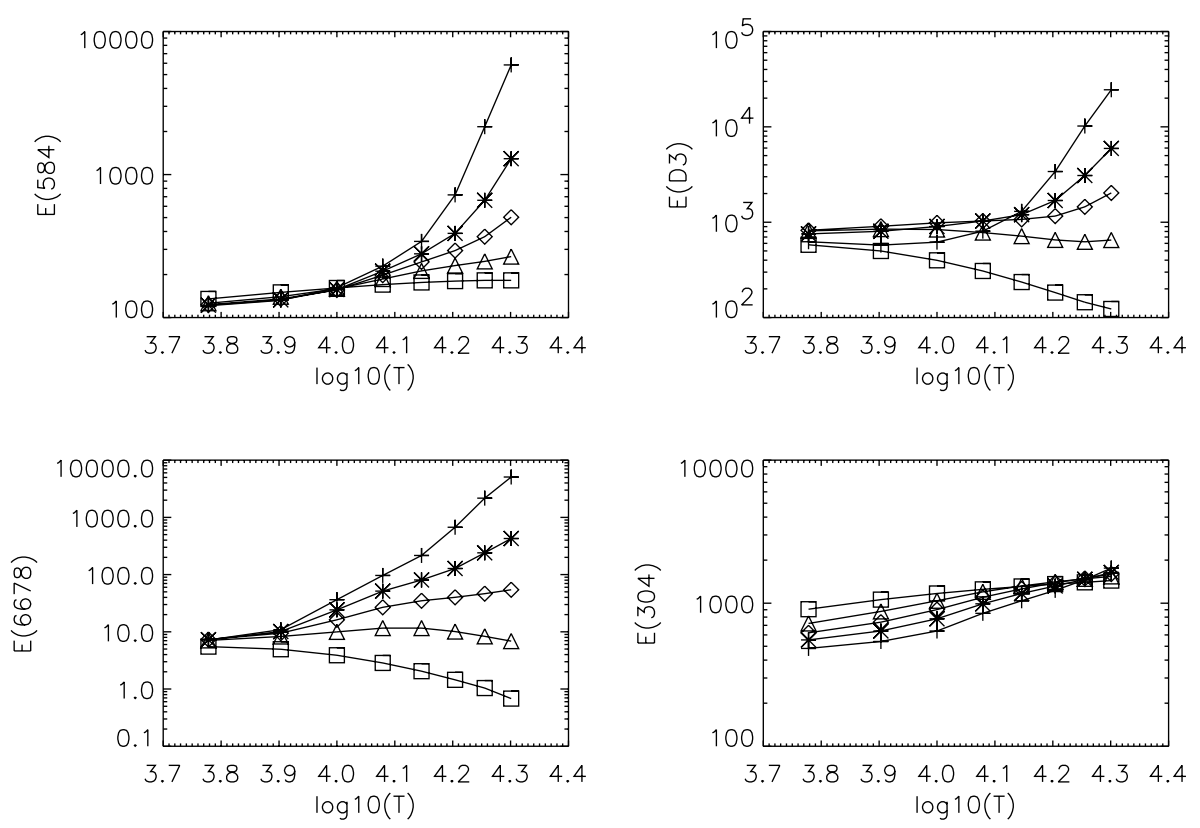

Fig. 9. Integrated intensities (in cgs units) for 4 lines as a function of the temperature computed for one slab width (1000 km) and five pressures: $0.02(\square), 0.05(\triangle), 0.1(\diamond), 0.2(*)$, and $0.5 \mathrm{dyn} / \mathrm{cm}^{2}(+)$.

$W=1000 \mathrm{~km}$. These two models are referenced in Table 5 and the hydrogen and electron mean population as well as the optical depths at the head of the different continua are given for each of the four abundances considered. The variation of helium mean populations, emergent profiles and integrated intensities with the helium abundance are represented in Figs. 13 to 15.

For the low temperature, low pressure model, the influence of the helium abundance is clearly visible on the neutral singlet states (Fig. 13, top panel). The excited singlet states mean populations are particularly enhanced with the abundance increase (by a factor greater than 6 as for the level 5). The triplet states and He II populations are less affected by the abundance. We can see from Table 5 that the increase of the He I ground state population leads to an optically thick resonance continuum, and this gives a decrease of the helium ionization with abundance. Thus the increase of abundance affects the population mechanism of neutral helium because of the increase of the optical depth at $504 \AA$ (and also a decrease of the optical depth at $912 \AA$ ). The optically thick lines are saturated and the 584 line emergent profile is slightly broadened by the abundance increase (optical depth effect), while the 304 line profile does not show any variation. The resulting integrated intensities are almost constant (Fig. 15 and four top panels of Fig. 14). The optically thin line profiles see their line center and line wings intensities enhanced by the abundance increase. The 6678 singlet line is the most affected: its integrated intensity is enhanced by a factor greater than 5 while the triplet D3 line integrated intensity is increased by a factor of less than 3 . These intensity enhancements for the optically thin lines with the helium abundance correspond to the increase of the upper state mean population of the related transition. The increase of the singlet-to-triplet line ratio with abundance is due to the fact that the optical depth of the 584 line grows with helium abundance. Thus, the emission of photons from the excited singlet states will be preferably through the optically thin lines (such as the 6678 line) rather than the resonance lines. In the triplet system the abundance effect is limited by the presence of the metastable level $1 \mathrm{~s} 2 \mathrm{~s}{ }^{3} \mathrm{~S}$.

For the high temperature, high pressure model, the evolution of the mean population densities is similar to the low temperature, low pressure case, but the excited states population increase is larger in this case (Fig. 13). Again, we observe a decrease of the helium ionization due to the increase of $\tau^{504}$. The increase of He abundance also raises the singlet-to-triplet line ratios (Fig. 15). The optically thin line profiles show the same evolution with abundance and again, there is a larger increase with abundance (of a factor more than 7) in the integrated intensity of the 6678 line than for the triplet line. The 304 line formation is still dominated by the scattering of the incident radiation (Fig. 14) and its integrated intensity is constant with the abundance variation. Finally, the 584 line exhibits a peak around $0.055 \AA$ from line center due to collisional excitation from the ground state. The height of this peak and its distance from the line center are increasing with the abundance.

From this study we see that the different sensitivities of line intensities (optically thick vs. optically thin, singlet vs. triplet) could be used, as well as the comparison with hydrogen lines, to improve the diagnostics of helium abundance in prominences. 
Table 5. Physical parameters for the two models considered for the abundance study. The hydrogen $\left(n_{\mathrm{H}}\right)$ and electron $\left(n_{\mathrm{e}}\right)$ densities are in $\mathrm{cm}^{-3} \cdot \tau^{912}, \tau^{504}$, and $\tau^{227}$ are the optical depth at the head of H I, He I and He II resonance continua.

\begin{tabular}{ccccc|ccccc}
\hline Model & $T$ & $P$ & $W$ & $n_{\mathrm{He}} / n_{\mathrm{H}}$ & $n_{\mathrm{H}}$ & $n_{e}$ & $\tau^{912}$ & $\tau^{504}$ & $\tau^{227}$ \\
\hline 6 & 6000 & 0.02 & \multirow{2}{*}{000} & 0.05 & $1.5+10$ & $8.9+9$ & $3.4+0$ & $4.4-1$ & $2.2-2$ \\
& & & & 0.10 & $1.4+10$ & $8.7+9$ & $3.3+0$ & $8.7-1$ & $3.8-2$ \\
& & & & 0.15 & $1.4+10$ & $8.5+9$ & $3.1+0$ & $1.3+0$ & $5.0-2$ \\
& & & & 0.20 & $1.3+10$ & $8.3+9$ & $3.0+0$ & $1.7+0$ & $6.0-2$ \\
\hline 99 & \multirow{2}{*}{18000} & \multirow{2}{*}{0.20} & \multirow{2}{*}{1000} & 0.05 & $3.9+10$ & $3.9+10$ & $4.5-1$ & $1.2+0$ & $5.1-2$ \\
& & & & 0.10 & $3.7+10$ & $4.0+10$ & $4.4-1$ & $2.4+0$ & $7.8-2$ \\
& & & & 0.15 & $3.6+10$ & $4.0+10$ & $4.3-1$ & $3.5+0$ & $9.8-2$ \\
& & & & 0.20 & $3.4+10$ & $4.0+10$ & $4.2-1$ & $4.5+0$ & $1.1-1$ \\
\hline
\end{tabular}
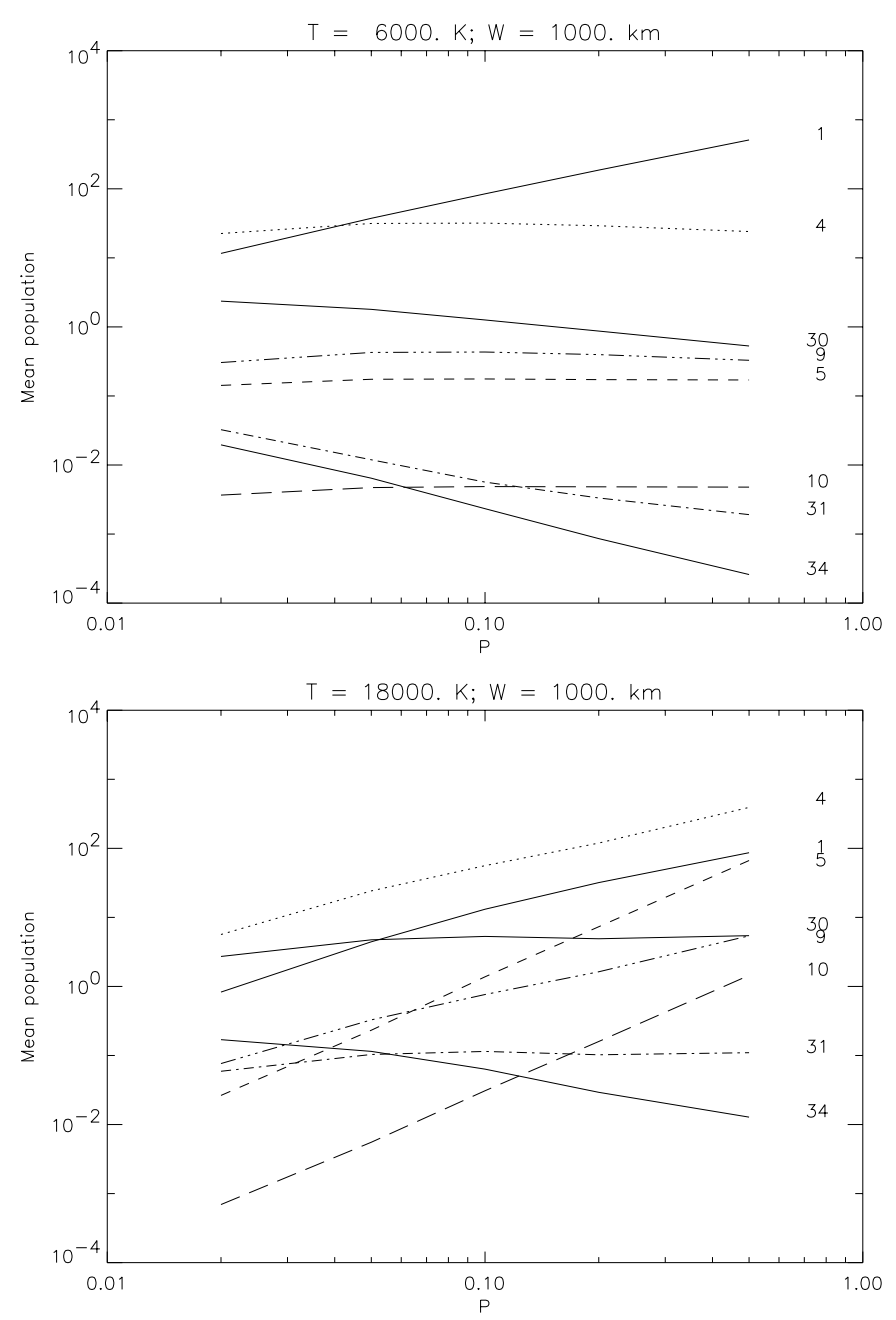

Fig. 10. Mean population densities $\left(\right.$ in $\mathrm{cm}^{-3}$ ) as a function of the pressure (in dyn $/ \mathrm{cm}^{2}$ ) for two different temperatures (6000 and $18000 \mathrm{~K})$ and one slab width $(1000 \mathrm{~km})$. Solid lines: ground states of He I (1), of He II (30), and He III continuum (34). The population densities for these levels are divided by $10^{8}$. Singlet excited levels represented: $1 \mathrm{~s} 2 \mathrm{p}{ }^{1} \mathrm{P}(5$, dashes $)$ and $1 \mathrm{~s} 3 \mathrm{~d}{ }^{1} \mathrm{D}$ (10, long dashes). Triplet levels are: $1 \mathrm{~s} 2 \mathrm{p}{ }^{3} \mathrm{P}(4$, dots) and $1 \mathrm{~s} 3 \mathrm{~d}{ }^{3} \mathrm{D}(9$, long dashes/dots). Level 31 is the $n=2$ level of He II (short dashes/dots).

\section{Correlations between lines}

It is interesting to look for theoretical correlations between different radiative or plasma properties such as the integrated intensities, optical depths, electron temperature, or gas pressure. This helps us to understand the physical processes that are at work in the line formation. Such a task has been done by Heinzel et al. (1994) for the hydrogen spectrum in 1D isothermal isobaric prominence models. Our aim here is to look for correlations between integrated intensities. For clarity we have restricted our study to only one value of the prominence thickness $(1000 \mathrm{~km})$.

\subsection{Helium triplet lines: $E(10830)$ and $E(D 3)$}

We present in Fig. 16 the relation between $E(10830)$ and $E$ (D3) for 40 models with a slab thickness of $1000 \mathrm{~km}$. This relation between these optically thin triplet lines is clearly linear. This linearity would only disappear in the case where at least one of the lines becomes optically thick and begins to saturate at line center. This may marginally happen for the 10830 line. In this case the ratio between the 10830 line intensity and another triplet line would slightly decrease. We stress that this linear relation is valid in the optically thin case for all the triplet lines we have studied. The only thing which varies from one line pair to another is of course the slope of the relation. This is due to the fact that in the triplet line source function the scattering term is dominant over the collisional term for a wide range of physical conditions. Thus the triplet line ratios mainly depend on the ratio of the incident line intensities. Here we obtain a constant ratio $E(10830) / E(D 3)=5.0$ for the 40 models represented in Fig. 16. In the following we will only consider the D3 line, keeping in mind that all the results presented for this line can easily be transposed to any other triplet line such as He I $\lambda \lambda 10830,7065,3889$, 3188 or $4471 \AA$ if the intensity ratio is known. Table 6 summarizes the different triplet line ratios for 40 models computed with $n_{\mathrm{He}} / n_{\mathrm{H}}=0.10$ and a slab thickness of $1000 \mathrm{~km}$. 

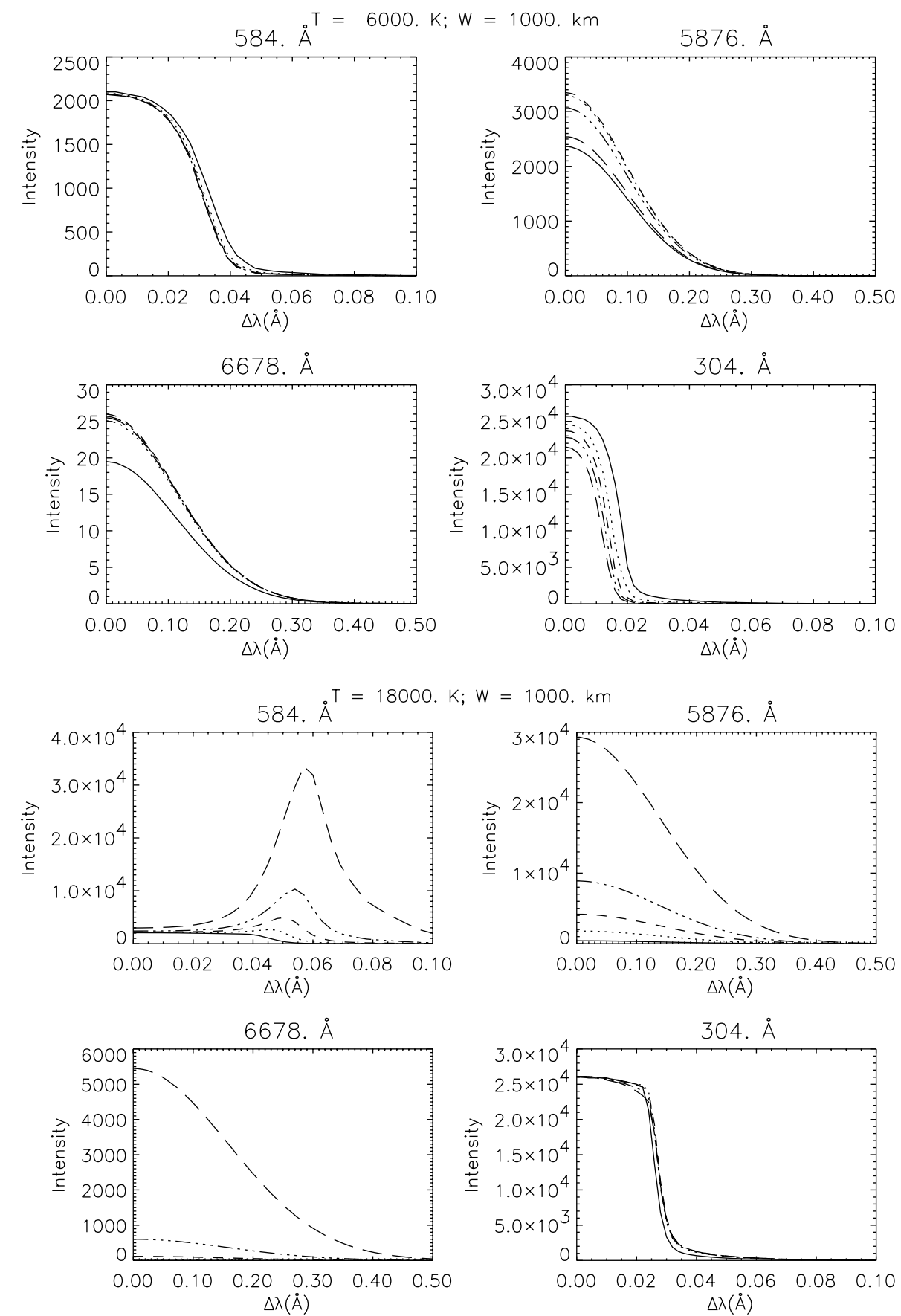

Fig. 11. Half emergent line profiles for one slab width $(1000 \mathrm{~km})$ at $6000 \mathrm{~K}$ (four top panels) and $18000 \mathrm{~K}$ (four bottom panels). Solid line: $P=0.02$; dots: $P=0.05$; short dashes: $P=0.1$; long dashes/dots: $P=0.2$; long dashes: $P=0.5$. Same units as in Fig. 5 .

\subsection{Singlet lines: $E(6678)$ and $E(584)$}

The relation between the optically thin He I $\lambda 6678 \AA$ and the optically thick resonance line He I $\lambda 584 \AA$ is represented in Fig. 17. These two lines have a common level $1 \mathrm{~s} 2 \mathrm{p}{ }^{1} \mathrm{P}$ which is the lower level of the 6678 transition (see Fig. 2). We can see that at low temperatures the 584 line is completely saturated and is independent of the model parameters (see Sect. 4.2) while the pressure variations decrease the 6678 line intensity. At a given temperature the line ratio depends on the pressure: an increase in the pressure leads to an increase of $E(6678) / E(584)$. This is particularly true at high temperatures where the collisional excitation plays an important role in the 584 line wing emission.

\subsection{Singlet and triplet lines: $E(6678)$ and $E(D 3)$}

The relation between optically thin singlet and triplet lines is not as simple as for the triplet-triplet case. We have in this case to consider how the helium statistical equilibrium is reached for the whole atom. In the 

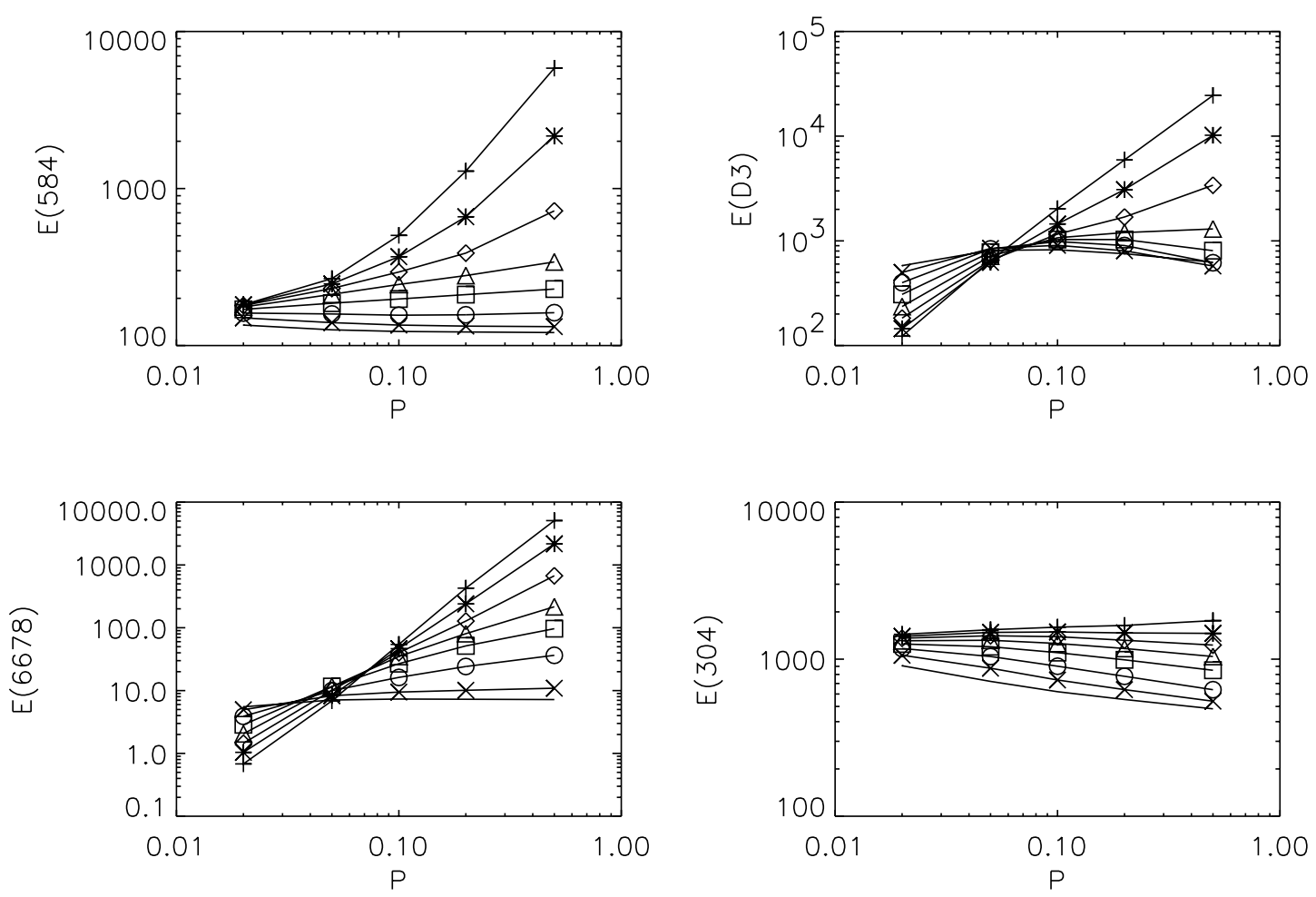

Fig. 12. Integrated intensities (in cgs units) for 4 lines as a function of the pressure computed for one slab width (1000 km) and 8 temperatures. Same symbols as in Fig. 6.

Table 6. He I triplet line ratios for 40 models with $W=$ $1000 \mathrm{~km}$.

\begin{tabular}{c|c}
\hline$E(10830) / E(\mathrm{D} 3)$ & 5.0 \\
$E(\mathrm{D} 3) / E(7065)$ & 7.5 \\
$E(\mathrm{D} 3) / E(3889)$ & 6.9 \\
$E(\mathrm{D} 3) / E(3188)$ & 20.6 \\
$E(\mathrm{D} 3) / E(4471)$ & 7.5 \\
\hline
\end{tabular}

singlet system the presence of the resonance lines provides an efficient way to deexcite the $1 \operatorname{snp}{ }^{1} \mathrm{P}$ states by spontaneous emission. In the triplet system the $1 \mathrm{~s} 2 \mathrm{~s}{ }^{3} \mathrm{~S}$ metastable level acts as a ground state. The intersystem collisions tend to drive the electrons from triplet to singlet states. Moreover we have already noticed in Sect. 4 the weakness of the singlet lines relative to the triplet lines. Figure 18 illustrates the pressure dependance of the relation between the singlet He I $\lambda 6678 \AA$ line integrated intensity and its triplet equivalent D3 line intensity. At low pressures $\left(0.02 \mathrm{dyn} / \mathrm{cm}^{2}\right)$ the relation between the two lines is almost linear. Landman \& Illing (1976) have found a similarly linear relation between the two lines for one observed prominence (see their Fig. 3). The observed range of intensities was smaller than that represented in our Fig. 18: the D3 maximum intensity was less than $3 \times 10^{3} \mathrm{erg} \mathrm{s}^{-1} \mathrm{~cm}^{-2} \mathrm{sr}^{-1}$, and the maximum 6678 intensity was about $50 \mathrm{erg} \mathrm{s}^{-1} \mathrm{~cm}^{-2} \mathrm{sr}^{-1}$. But we can see that if the pressure is higher the relation between the two lines is no longer linear at all. At $0.05 \mathrm{dyn} / \mathrm{cm}^{2}$ the integrated intensities are almost constant whatever the temperature, giving a limit ratio. If we increase the pressure this same limit value for the line ratio will be found for the lower temperatures. In this situation the formation of the two lines is mainly due to the scattering of the incident radiation because of a high optical thickness in the incident continuum radiation. For high pressure, high temperature models, the same optical depth is reduced and collisional excitation becomes important: this gives an increasing ratio of the two lines with the temperature and the pressure.

\section{4. $E(H e I I \lambda 304)$ and $E(H e I \lambda 584)$}

Ionized helium is coupled to neutral helium in several different ways in our model atom. Radiative bound - free transitions (photoionization and collisional ionization) are allowed between all neutral helium states and the ground state of He II. The photoionization of the He I ground level plays an important role since it is the main way to populate the excited levels by recombinations (PR process) when the temperature is not high enough to produce significant collisional excitation from the ground level towards the triplet states.

The relation between He II $\lambda 304 \AA$ and He I $\lambda 584 \AA$ shown in Fig. 19 indicates a decreasing ratio $E(304) / E(584)$ with the temperature: the 304 line intensity also grows with the temperature, but more slowly than the 584 line intensity. At high temperatures the 584 line emission increases with the pressure because of collisional 

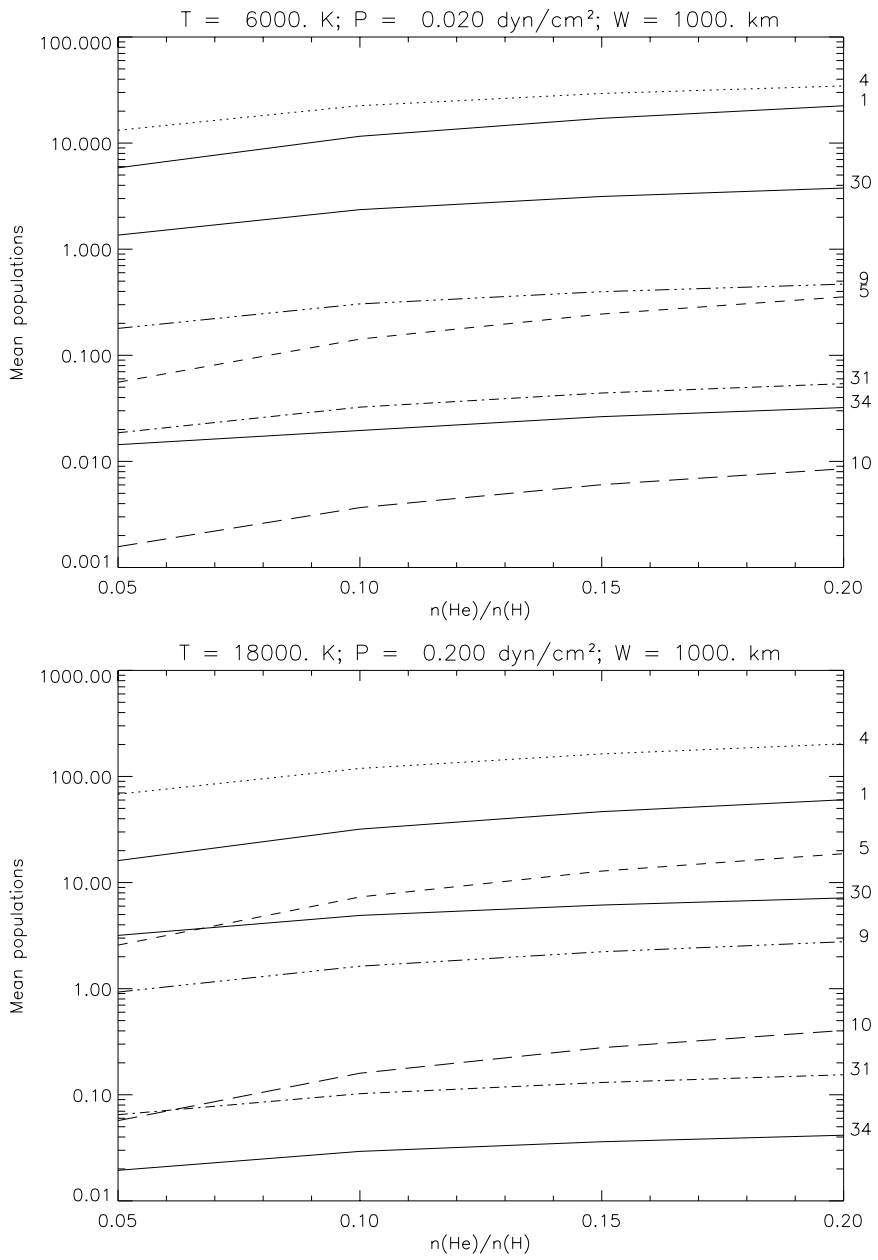

Fig. 13. Mean population densities $\left(\right.$ in $\mathrm{cm}^{-3}$ ) as a function of the helium abundance for two different models: $T=6000 \mathrm{~K}$, $P=0.02 \mathrm{dyn} / \mathrm{cm}^{2}$ and $W=1000 \mathrm{~km}$ (top panel); $T=$ $18000 \mathrm{~K}, P=0.2 \mathrm{dyn} / \mathrm{cm}^{2}$ and $W=1000 \mathrm{~km}$ (bottom panel). Solid lines: ground states of He I (1), of He II (30), and He III continuum (34). The population densities for these levels are divided by $10^{8}$. Singlet excited levels represented: $1 \mathrm{~s} 2 \mathrm{p}{ }^{1} \mathrm{P}$ (5, dashes) and $1 \mathrm{~s} 3 \mathrm{~d}{ }^{1} \mathrm{D}$ (10, long dashes). Triplet levels are: $1 \mathrm{~s} 2 \mathrm{p}{ }^{3} \mathrm{P}(4$, dots $)$ and $1 \mathrm{~s} 3 \mathrm{~d}{ }^{3} \mathrm{D}(9$, long dashes/dots). Level 31 is the $n=2$ level of HeII (short dashes/dots).

excitation while the 304 line is saturated and is formed by scattering of the incident radiation. A decrease of the temperature increases the $E(304) / E(584)$ ratio up to a limit value which corresponds to a situation where both lines are formed by the scattering of the incident radiation.

\subsection{HeI $D 3$ and $H \beta$ lines}

The intensity ratio between the helium D3 line and the hydrogen Balmer lines has been measured by several observers (Landman \& Illing 1976, 1977; Landman et al. 1977; Stellmacher 1979; Stellmacher \& Wiehr 1995, 1997; de Boer et al. 1998, for instance). Figure 20 shows the theoretical behaviour of the $E(\mathrm{D} 3) / E(\mathrm{H} \beta)$ ratio with the different physical parameters already considered in this work. First we can see that this ratio decreases with the slab thickness. $E(D 3)$ increases with the slab thickness (Fig. 6), but more slowly than the $\mathrm{H} \beta$ intensity. $E(\mathrm{D} 3) / E(\mathrm{H} \beta)$ is not very sensitive to the temperature at low pressure. We can see that at a fixed temperature the increase of the pressure causes the ratio to decrease because of a decrease of the mean helium ionization ratio. For the highest pressures where the ionization is low the only way to observe a significant $E(\mathrm{D} 3) / E(\mathrm{H} \beta)$ value is to have high temperatures. The evolution of the ratio with helium abundance reflects the evolution of the D3 intensity with $n_{\mathrm{He}} / n_{\mathrm{H}}$ (see Fig. 15) but with a higher slope. An increase of a factor of 4 in helium abundance leads to an increase of a factor of 3 for the intensity ratio in the case of a low pressure, low temperature model ( $\diamond$ in Fig. 20, bottom right panel). This corresponds to a decrease of about $15 \%$ of the $\mathrm{H} \beta$ intensity with the abundance increase.

Heasley \& Milkey (1976) have already discussed (see their Fig. 1) the theoretical behaviour of this relation relative to temperature, pressure, and column mass. If we increase the total column mass (increase of the $\mathrm{H} \beta$ intensity) the prominence becomes optically thick in the Lyman continuum and the excitational and ionizing radiation penetrates less deeply in the slab, thus decreasing the ionization of helium. An increase in the pressure has the same effect: we obtain an increase of the recombination rates, thus reducing the helium ionization. Finally, a decrease in the temperature leads to a decrease of hydrogen and helium ionization (or an increase of the Lyman continuum optical thickness). We know that the recombination from the He II ground state is the main mechanism which populates the helium triplet states. This is consistent with the photoionization-recombination $(\mathrm{PR})$ scheme which is believed to be the main population mechanism for helium in the solar atmosphere for temperatures below $20000 \mathrm{~K}$ (see e.g., Andretta \& Jones 1997). These effects are also seen in our Fig. 3 (see Sect. 3.2). The tendancy of a lowering of the curve with increasing $\mathrm{H} \beta$ intensities is also visible in the Fig. 6 of, e.g., de Boer et al. (1998). Our conclusion about helium excitation and ionization conditions are that as the hydrogen density increases, the $n_{\mathrm{HeII}} / n_{\mathrm{He} \text { I }}$ ratio decreases, but more rapidly at slab center where there is much less UV continuum radiation. This produces a too weak ionization at slab center to populate helium triplet states.

\section{Conclusion}

In this work we have presented new non-LTE computations of the helium emerging spectrum in a onedimensional isothermal isobaric prominence. We use a $29+4+1$ levels model for the helium atom and PRD for the hydrogen $\operatorname{Ly} \alpha, \operatorname{Ly} \beta$, and the first resonance lines of He I and He II. Detailed incident profiles were used for the principal transitions. This approach leads to some differences with the previous calculations carried out by Heasley et al. (HMP; HM3) who used CRD and frequencyindependent incident line profiles for the helium transitions, and only 15 bound levels for the neutral helium atom. The theoretical study of Sects. 4 and 5 has shown 

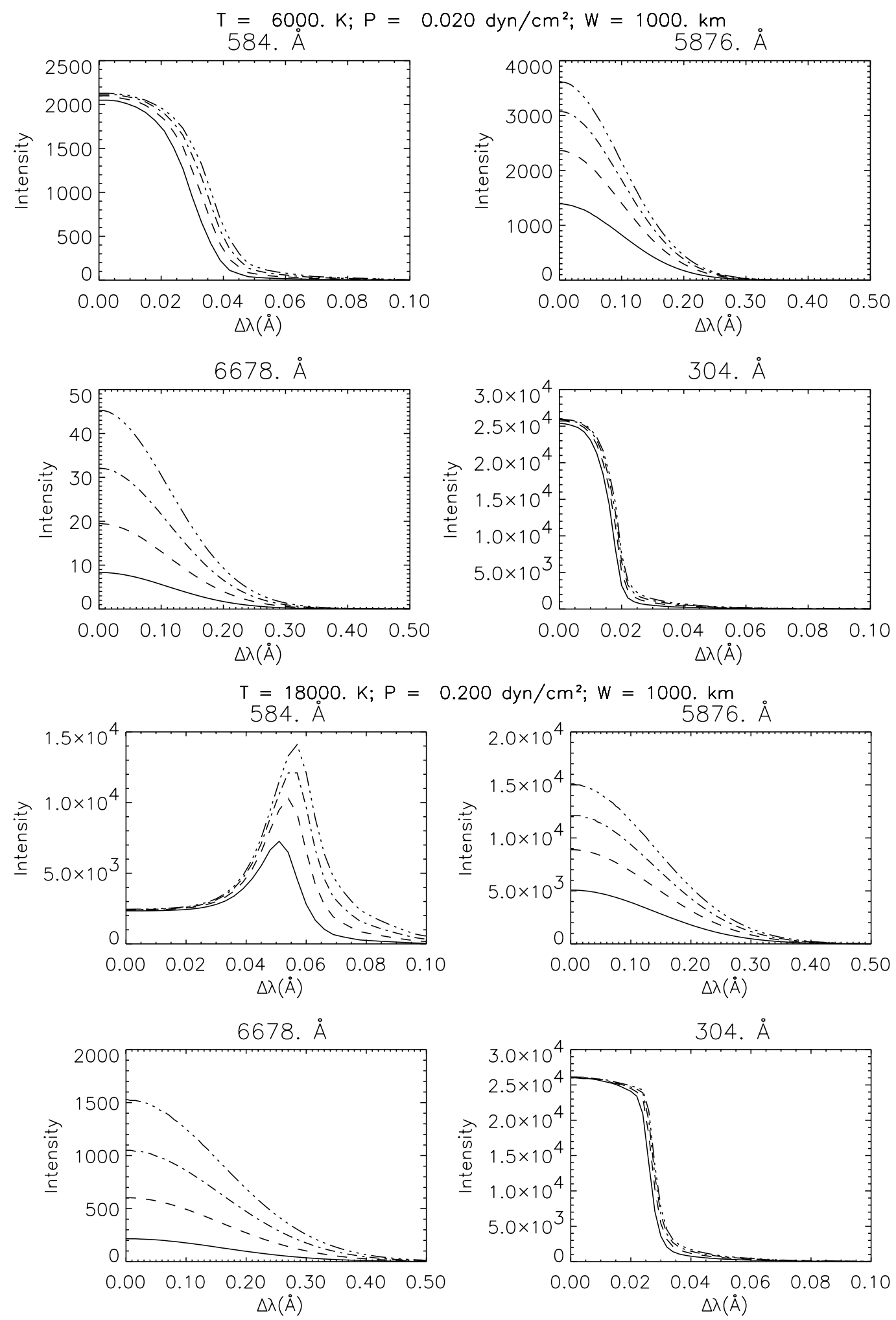

Fig. 14. Half emergent line profiles for two different models: $T=6000 \mathrm{~K}, P=0.02 \mathrm{dyn} / \mathrm{cm}^{2}$ and $W=1000 \mathrm{~km}$ (four top panels); $T=18000 \mathrm{~K}, P=0.2 \mathrm{dyn} / \mathrm{cm}^{2}$ and $W=1000 \mathrm{~km}$ (four bottom panels). Solid line: $n_{\mathrm{He}} / n_{\mathrm{H}}=0.05$; short dashes: $n_{\mathrm{He}} / n_{\mathrm{H}}=0.10 ;$ dots/dashes: $n_{\mathrm{He}} / n_{\mathrm{H}}=0.15 ;$ long dots/dashes: $n_{\mathrm{He}} / n_{\mathrm{H}}=0.20$. Same units as in Fig. 5 .

the different effects of the physical parameters of our computations on the mean populations of several helium states and on the emitted intensities. We particularly noticed the different behaviour of the singlet and of the triplet helium lines which can be useful for the prominence plasma diagnostic. The presence of the resonance lines in the singlet system is of primary importance for the formation of the neutral helium emerging spectrum. We have also shown that, in the range of temperatures and pressures we considered, the He II $\lambda 304 \AA$ line is formed only by the 

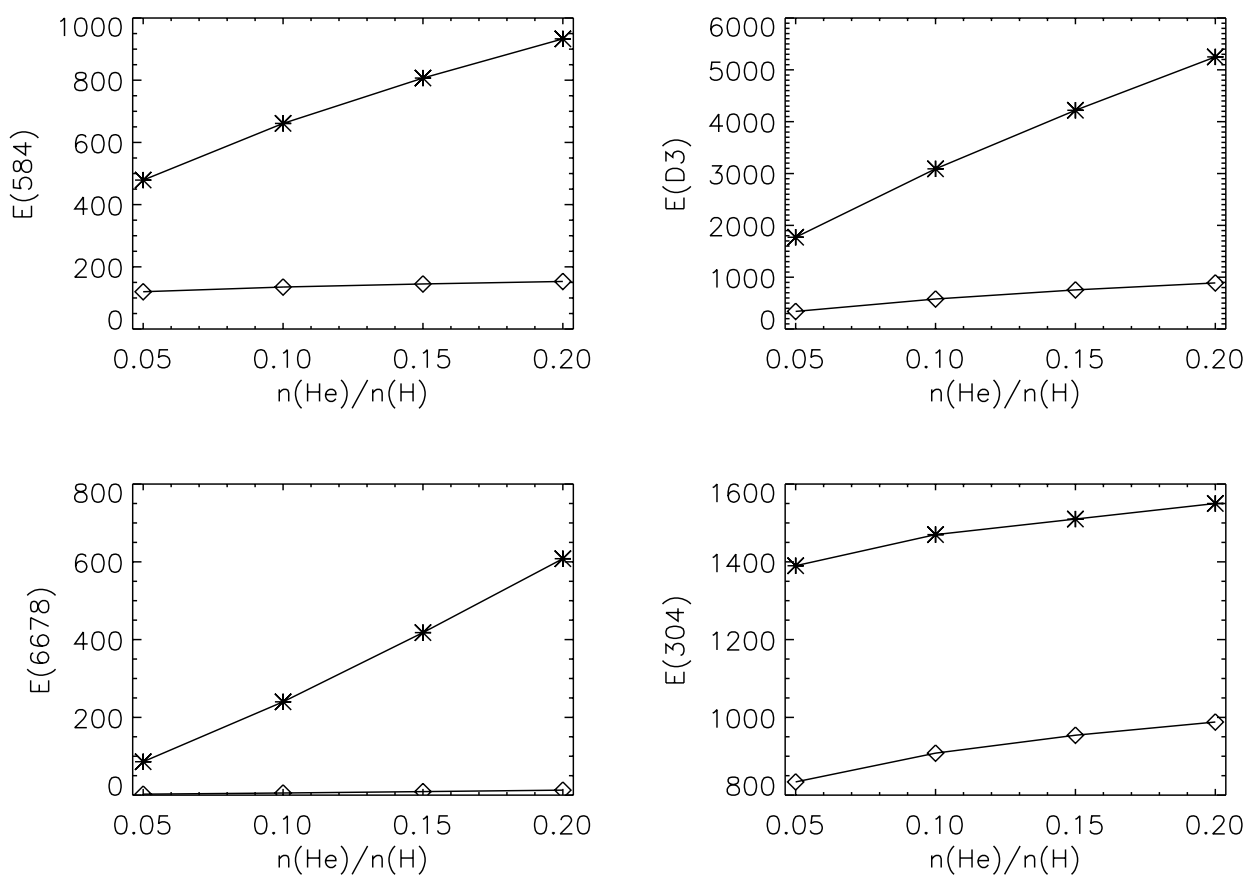

Fig. 15. Integrated intensities (in cgs units) for 4 lines as a function of the abundance computed for 2 models: $T=6000 \mathrm{~K}$, $P=0.02 \mathrm{dyn} / \mathrm{cm}^{2}$ and $W=1000 \mathrm{~km}(\diamond) ; T=18000 \mathrm{~K}, P=0.2 \mathrm{dyn} / \mathrm{cm}^{2}$ and $W=1000 \mathrm{~km}(*)$.

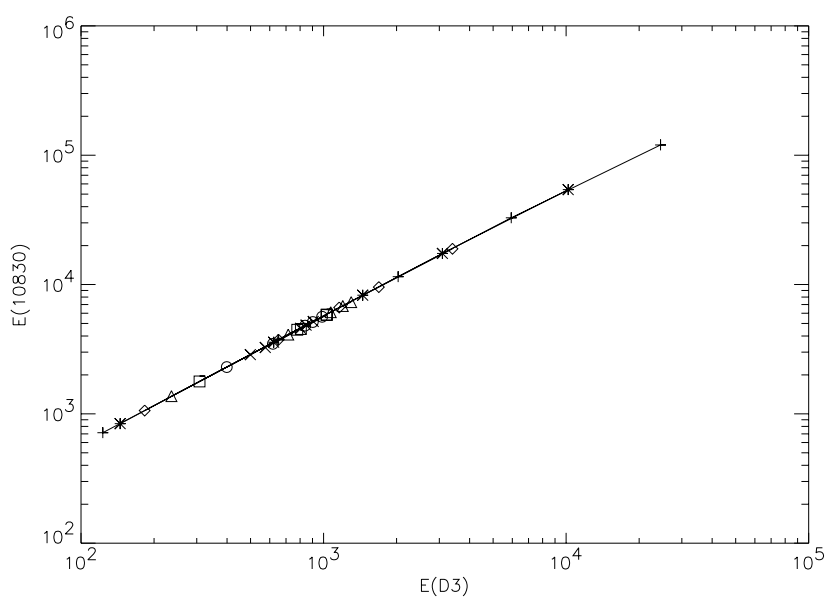

Fig. 16. $E($ He I $\lambda 10830)$ versus $E(D 3)$. Integrated intensities are in cgs units. Computations are made for 5 pressures and 8 temperatures. Same symbols as in Fig. 6.

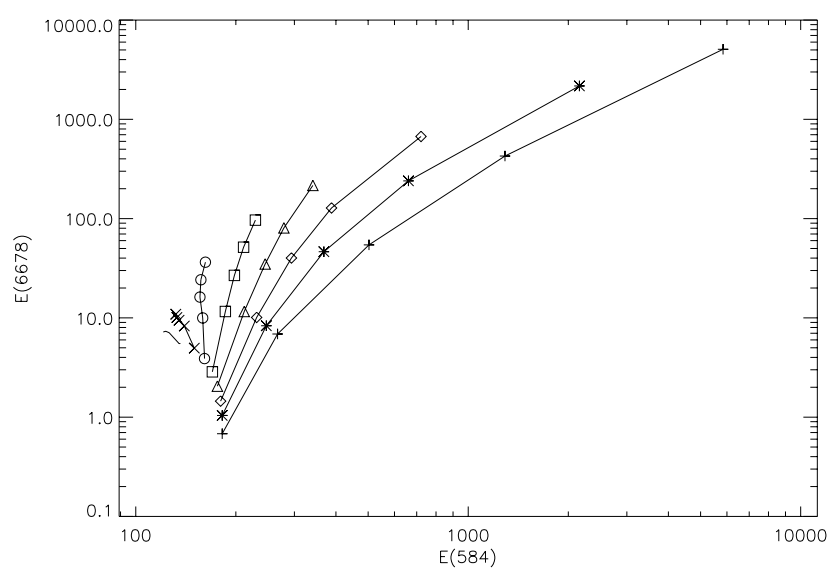

Fig. 17. $E($ He I $\lambda 6678)$ versus $E($ He I $\lambda 584)$ for 8 temperatures. Same symbols as in Fig. 6.

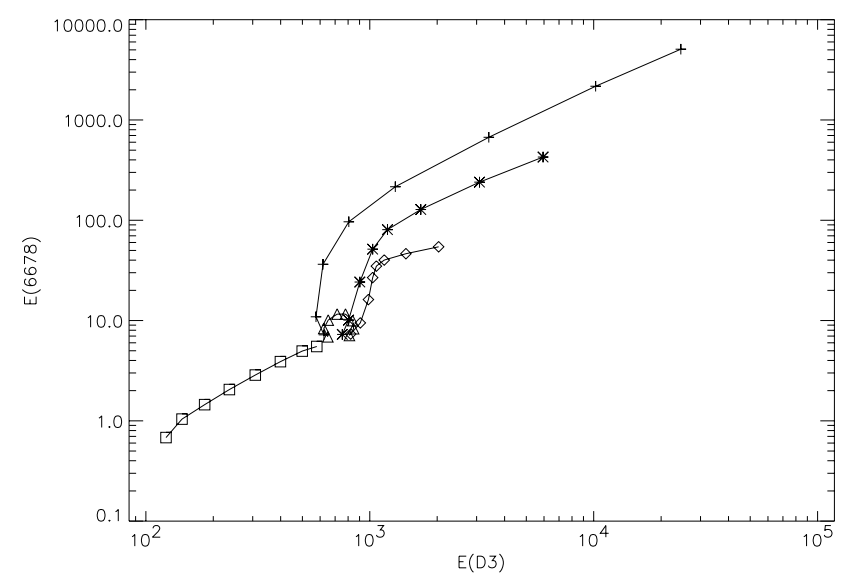

Fig. 18. $E($ He I $\lambda 6678)$ versus $E(D 3)$ for 5 pressures. Same symbols as in Fig. 9.

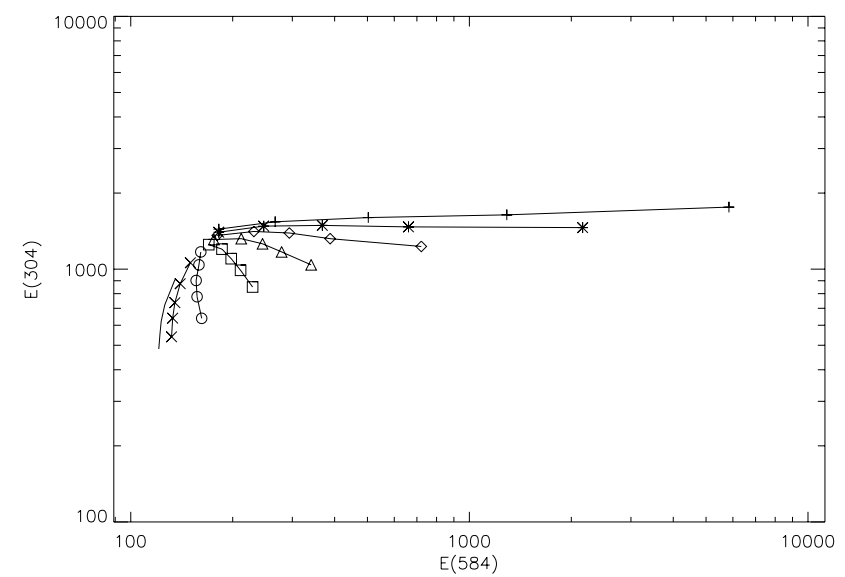

Fig. 19. $E($ He II $\lambda 304)$ versus $E($ He I $\lambda 584)$ at 8 temperatures. Same symbols as in Fig. 6. 

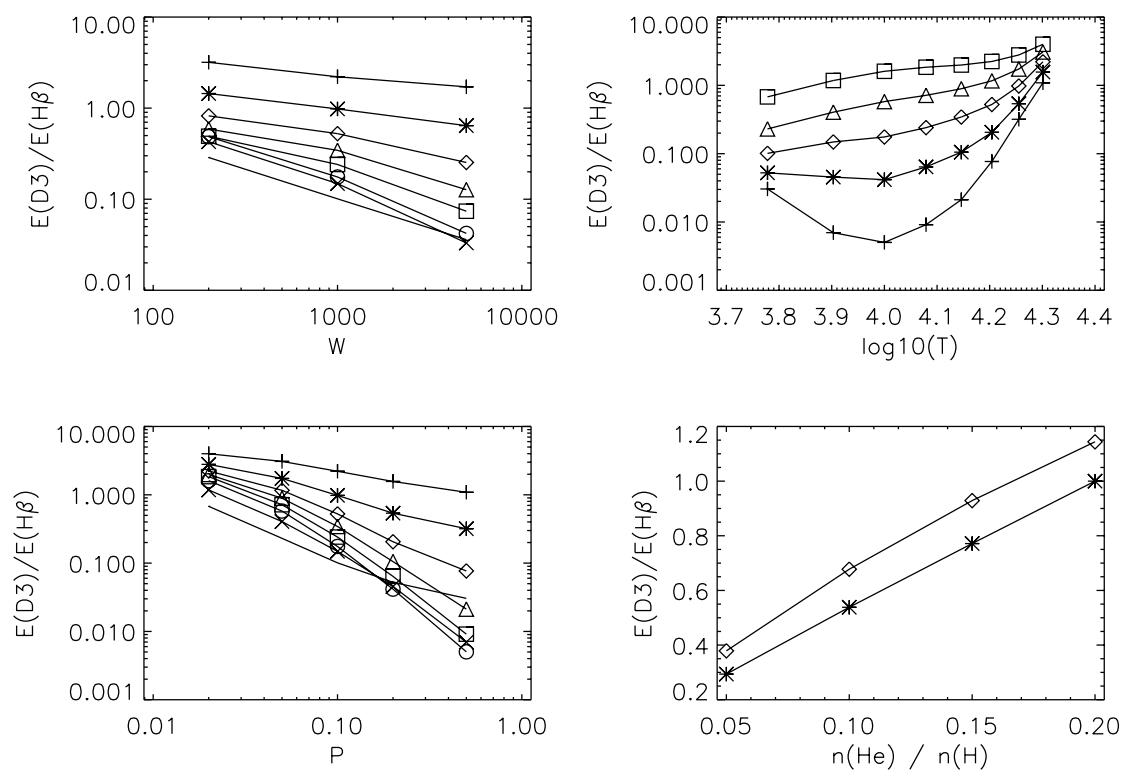

Fig. 20. $E(\mathrm{D} 3) / E(\mathrm{H} \beta)$ as a function of: the slab width (top left panel) at 8 temperatures (same symbols as in Fig. 6); the temperature (top right panel) at 5 pressures (same symbols as in Fig. 9); the pressure (bottom left panel) at 8 temperatures (same symbols as in Fig. 6); and the helium abundance (bottom right panel) for 2 models (same symbols as in Fig. 15).

scattering of the incident radiation. The number of levels in the He II ion that we have taken into account allows the study of the formation of this resonance line. In a next paper we will compare our isothermal, isobaric computations for the EUV helium lines with observations obtained with the SUMER instrument onboard of SoHO.

Acknowledgements. We wish to thank J.-C. Vial for reading the manuscript, and T. Holzer for his encouraging comments on this work. Computations are performed on a IBM RS/6000 scalar computer at the Multi-Experiment Data Operation Centre for SoHO (IAS) and at the Institut du Développement et des Ressources en Informatique Scientifique.

\section{References}

Aggarwal, K. M., Berrington, K. A., Kingston, A. E., \& Pathak, A. 1991, J. Phys. B, 24, 1757

Aggarwal, K. M., Callaway, J., Kingston, A. E., \& Unnikrishnan, K. 1992, ApJS, 80, 473

Allen, C. W. 1973, Astrophysical quantities, 3ème édition (London: University of London, Athlone Press)

Andretta, V., \& Jones, H. P. 1997, ApJ, 489, 375

Andretta, V., Kucera, T. A., \& Poland, A. I. 1999, in Solar and Stellar Activity: Similarities and Differences, ASP Conf. Ser., 158, 162

Auer, L. H., \& Mihalas, D. 1970, MNRAS, 149, 65

Auer, L. H., \& Mihalas, D. 1973, ApJS, 25, 433

Benjamin, R. A., Skillman, E. D., \& Smits, D. P. 1999, ApJ, 514, 307

Benson, R. S., \& Kulander, J. L. 1972, Sol. Phys., 27, 305

de Boer, C. R., Stellmacher, G., \& Wiehr, E. 1998, A\&A, 334, 280

Dimitrijevic, M. S., \& Sahal-Brechot, S. 1984, JQSRT, 31, 301

Feautrier, P. 1964, C. R. Acad. Sci. Paris, 258, 3189

Fernley, J. A., Seaton, M. J., \& Taylor, K. T. 1987, J. Phys. B, 20, 6457
Gouttebroze, P., Heinzel, P., \& Vial, J.-C. 1993, A\&AS, 99, 513

Gouttebroze, P., \& Labrosse, N. 2000, Sol. Phys., 196, 349

Griem, H. R. 1974, Spectral line broadening by plasmas (Pure and Applied Physics, New York: Academic Press)

Heasley, J.-N., \& Mihalas, D. 1976, ApJ, 205, 273

Heasley, J.-N., Mihalas, D., \& Poland, A.-I. 1974, ApJ, 192, 181

Heasley, J.-N., \& Milkey, R.-W. 1976, ApJ, 210, 827

Heasley, J.-N., \& Milkey, R.-W. 1978, ApJ, 221, 677

Heasley, J.-N., \& Milkey, R.-W. 1983, ApJ, 268, 398

Heinzel, P., Gouttebroze, P., \& Vial, J.-C. 1987, A\&A, 183, 351

Heinzel, P., Gouttebroze, P., \& Vial, J.-C. 1994, A\&A, 292, 656

Heroux, L., Cohen, M., \& Higgins, J. E. 1974, J. Geophys. Res., 79,5237

Hirayama, T. 1971, Sol. Phys., 19, 384

Kucera, T. A., Andretta, V., \& Poland, A. I. 1998, Sol. Phys., 183, 107

Landman, D. A., Edberg, S. J., \& Laney, C. D. 1977, ApJ, 218,888

Landman, D. A., \& Illing, R. M. E. 1976, A\&A, 49, 277

Landman, D. A., \& Illing, R. M. E. 1977, A\&A, 55, 103

Li, K., Gu, X., \& Chen, X. 2000, MNRAS, 313, 761

Mariska, J. T., Doschek, G. A., \& Feldman, U. 1979, ApJ, 232, 929

Mihalas, D., \& Stone, M. E. 1968, ApJ, 151, 293

Morozhenko, N. N. 1984, Sol. Phys., 92, 153

Stellmacher, G. 1979, Sol. Phys., 61, 61

Stellmacher, G., \& Wiehr, E. 1995, A\&A, 299, 921

Stellmacher, G., \& Wiehr, E. 1997, A\&A, 319, 669

Wiese, W. L., Smith, M. W., \& Glennon, B. M. 1966, Atomic transition probabilities, Hydrogen through Neon. A critical data compilation (NSRDS-NBS 4, Washington, D.C.: US Department of Commerce, National Buereau of Standards)

Yakovkin, N. A., Zeldina, M. Y., \& Lhagvazhav, C. 1982, Sol. Phys., 81, 339 